https://doi.org/10.5719/aub-g/70.1/7

\title{
LA VÉGÉTATION MÉDITERRANÉENNE ARIDE DANS LES AIRES DE TRANSITION : TYPOLOGIE, RICHESSE ET DÉFIS DE VALORISATION. CAS DES MONTS DE MATMATA (SUD TUNISIEN)
}

\author{
ALI HANAFI ${ }^{1}$, OUMAYMA ARIDHI ${ }^{2}$
}

Abstract

The Arid Mediterranean vegetation in transition areas: typology, richness and challenges of its valorization. Case of Matmata mountains (south of Tunisia): The mountains of Matmata constitute a natural region of southeastern Tunisia, which constitutes a transition between the Mediterranean environment and the desert one, and which is characterized by the presence of a singular plant unit formed by a Mediterranean scrubland located within a landscape steppe. The objective of this work is to show the specific diversity of this vegetation as well as the ways of its development. For this, vegetation mapping was established following the completion of around sixty field surveys, which underwent multivariate analyses (CFA, HAC) in order to determine the plant groups in place. The results showed a significant diversity of these groups, as well as a rich flora characterized by the presence of several endemic or rare species. This is the case with Rosmarinus officinalis var. troglodytarum and Teucrium alopecurus now threatened by degradation and which require special attention not only from environmentalists but also from investors. Indeed, this scrubland could be integrated into eco-tourist circuits. In addition, several of its species could be exploited for medicinal uses, which could allow the villages of Matmata to boost their commercial activities and limit their economic isolation.

Keywords: Scrubland - Steppe vegetation - Vegetation survey - Diversity Matmata mountain- South-East Tunisia.

${ }^{1}$ Biogéographe (PhD), Enseignant-chercheur, Labo. GEOMAG, FLAH-Univ. de Manouba, TUNISIE, E-mail : ali.hanafi@flah.uma.tn

2 Biogéographe, Doctorante, Labo. GEOMAG, FLAH-Univ. de Manouba, TUNISIE, E-mail: aridhi.oumayma@gmail.com 


\section{Résumé}

Les monts de Matmata constituent une région naturelle du sud-est tunisien qui constitue une transition entre le milieu méditerranéen et celui désertique, et qui se caractérise par la présence d'une unité végétale singulière formée par un matorral méditerranéen localisé au sein d'un paysage steppique. L'objectif de ce travail est de montrer la diversité spécifique de cette végétation ainsi que les voies de sa valorisation. Pour ceci, une cartographie de la végétation a été établie suite à la réalisation d'une soixantaine de relevés de terrain et qui ont subi des analyses multivariées (AFC, CAH) afin de déterminer les groupements végétaux en place. Les résultats ont montré une importante diversité des groupements présents, ainsi qu'une richesse floristique caractérisée par la présence de plusieurs espèces endémiques ou rares. C'est le cas de Rosmarinus officinalis var. troglodytarum et du Teucrium alopecurus ${ }^{3}$ aujourd'hui menacées par la dégradation et qui nécessitent une attention particulière non seulement de la part des écologistes mais aussi des entrepreneurs. En effet, ce matorral pourrait être intégré dans les circuits éco-touristiques. Par ailleurs, plusieurs de ses espèces peuvent être valorisés pour les usages médicinaux, ce qui pourrait permettre aux villages de Matmata de dynamiser leurs activités commerciales et de limiter leur enclavement économique.

Mots-clés : Matorral - Végétation steppique - Relevé de végétation - Diversité Matmata-Sud-Est Tunisien.

\section{Introduction}

Les régions arides tunisiennes se caractérisent par la richesse de leurs ressources naturelles. Celles-ci sont de plus en plus dégradées sous l'effet des contraintes naturelles et surtout sous l'effet d'une pression humaine accrue. Les écosystèmes steppiques de ces régions présentent le plus souvent un équilibre fragile qui, lorsqu'il a été rompu, a laissé la place à des paysages désertifiés (Floret et al 1978, Floret et Pontanier 1982...). Dans plusieurs cas ces équilibres naturels rompus ont été remplacés par un équilibre artificiel basé sur un lourd dispositif d'aménagements de rétention des eaux et des sols et sur une présence humaine quasi continue pour les maintenir. Aujourd'hui et malgré l'effort des différents acteurs (Etat, collectivités locales, habitants...) pour freiner la dégradation des écosystèmes, l'évolution des modes d'occupation de sol, la pression croissante et continue sur les ressources naturelles dont le désir est d'améliorer les revenus familiaux associés au stress climatique,

\footnotetext{
${ }^{3}$ La nomenclature des espèces végétales adoptée dans ce travail se réfère aux travaux de
} Le Floc'h et al 2010. 
ont entraîné des modifications profondes des paysages et des systèmes écologiques. Ces modifications ont essentiellement touché la végétation steppique qui a connu une fragmentation spatiale, une baisse de son recouvrement et de sa diversité et un changement de sa composition floristique (Hanafi 2008).

Certaines unités de cette végétation constituent des formations originales renfermant une importante richesse spécifique et écosystémique. C'est le cas dans les monts de Matmata du matorral méditerranéen azonal à Juniperus phoenicea, Rosmarinus officinalis var. troglydatrum et Stipa tenacissima. Cette formation caractéristique des montagnes, comporte une diversité de milieux physiques et écologiques, avec un climat de type méditerranéen aride. L'empreinte humaine y est ancienne et la dégradation des écosystèmes est très poussée. Malgré cette situation, ce matorral constitue aujourd'hui un patrimoine naturel qui nécessite d'être conservé, mais aussi une potentialité à valoriser pour maintenir les équilibres naturels et économiques dans cette région. C'est dans ce cadre que ce travail a été entrepris dans L'objectif de cartographier cette végétation originale, d'évaluer sa richesse spécifique et écosystémique et d'étudier les possibilités de sa valorisation éco-touristique et médicinale afin de participer au désenclavement économique des monts de Matmata. L'intérêt est de savoir comment la gouvernance et la gestion des ressources végétales de Matmata peut représenter non seulement une meilleure conservation de leur diversité mais aussi de leur durabilité ?

\section{Zone d'étude}

La zone d'étude fait partie de la Tunisie méridionale. Elle s'étend sur la partie centrale des monts de Matmata entre le village de Toujène à $33^{\circ} 28^{\prime} \mathrm{N}$ et $10^{\circ} 07^{\prime} \mathrm{E}$ et la ville de Béni Khédache à $33^{\circ} 15^{\prime} \mathrm{N}$ et $10^{\circ} 11^{\prime} \mathrm{E}$ couvrant ainsi une superficie totale d'environ 54.000 ha (Fig. 1).

Cette zone de montagne s'étale sur une première partie de la délégation de Dkhilet Toujène rattaché au gouvernorat de Gabès et une deuxième partie de la délégation de Béni Khédache rattachée au gouvernorat de Médenine. 


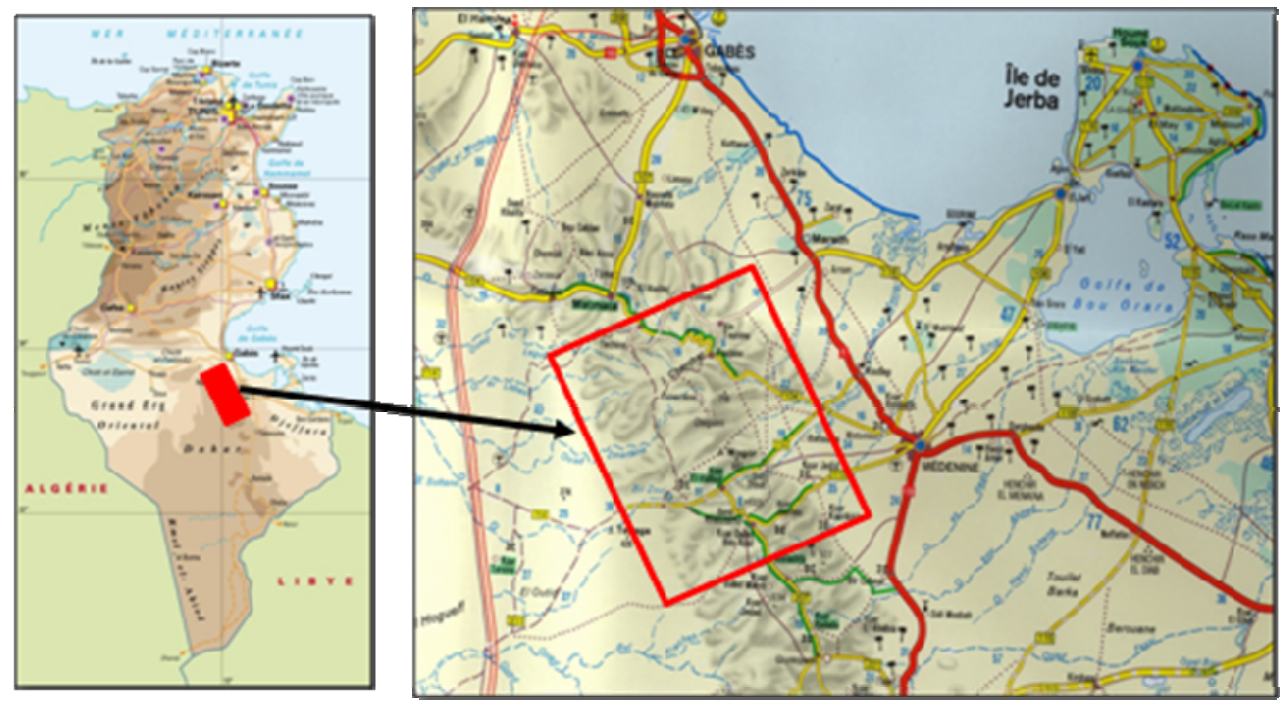

Fig. 1. Localisation de la zone d'étude

\section{Le relief et les sols}

La zone d'étude est essentiellement dominée par un paysage de montagnes. Bien visibles avec un escarpement fort, ces montagnes constituent un alignement d'une orientation générale ENW-WSE. L'altitude moyenne de la crête est d'environ $500 \mathrm{~m}$ avec des sommets de $682 \mathrm{~m}$ à Mzenzen et $648 \mathrm{~m}$ à Moggor (Hanafi 2008). L'ensemble des montagnes forme une cuesta intégrant une partie des monts de Matmata et correspond à l'extrémité septentrionale de la chaîne du Dahar. Cette unité est partout découpée par des vallées dominées par un matériel tendre, le plus souvent limoneux ou marneux (Ben Fredj 2012). En face du front de la cuesta regardant l'Est, s'étale un ensemble de petites montagnes et de collines scellées par une croûte calcaire tel que Tebaga et Zemlet El Bhim. A la surface du plateau prédominent des roches dures représentées essentiellement par les calcaires et les dolomies rencontrés dès le Permien jusqu'au complexe Mio-Plio-Quaternaire (Ben Fredj, 2012). Les sols dominants ici sont des minéraux bruts, souvent érodés et très peu profonds reposant sur une roche dure (dolomies) ou sur la croûte calcaire (Taamallah et Ben Kehia 2006). 
En bas des montagnes s'étendent des piémonts étroits avec des altitudes entre $200 \mathrm{~m}$ et $300 \mathrm{~m}$ et une pente ne dépassant pas $4 \%$. D'après Ben Fredj (2012), les héritages quaternaires dans cette région sont caractérisés par une variété de formes allant des cônes de déjection à gros éboulis aux terrasses en passant par des glacis d'érosion. Les dépôts de pente sont généralement cimentés par une croûte calcaire. Par ailleurs, les interfluves et les ravins sont colmatés par des argiles et des limons sableux à nodules calcaires d'âge Messinien et appelée communément les «loss de Matmata ». Ce sont ces limons qui constituent les paléosols de Matmata accumulés sur des épaisseurs souvent remarquables (Coudé-Gaussen 1991, Mtimet 1994). Cette formation est à plusieurs endroits scellée par une croûte calcaire du pléistocène inférieur (Ben Fredj 2012).

A l'est de la zone s'étend une plaine étroite de quelques kilomètres de large et qui constitue le début de la vaste plaine de la Jeffara. Son altitude est comprise entre $100 \mathrm{~m}$ et $200 \mathrm{~m}$ et est orientée NW-SE. Le substrat en place est dominé par des formations néogènes (Mio-pliocène) d'argiles sableuses à gypseuses (Ben Kehia et al 2002). Cette unité topographique se caractérise par une terrasse fluviale visible le long des oueds qui descendent des montagnes, et qui est formée par un dépôt grossier composé de galets hétérométriques moyennement émoussés et de blocs provenant directement des terrains crétacés des monts de Matmata (Ben Fredj 2012). Les sols dominants sont généralement peu évolués et isohumiques composés de calcaires et de limons souvent tronqués ou couverts par des encroûtements calcaires ou gypseux (Taamallah et Ben Kehia 2006). Leur pauvreté et leur remaniement continu par les agents de l'érosion éolienne et hydrique sont favorisés par l'aridité du climat et par la faible couverture végétale.

\section{Climat et ressources en eau}

La zone d'étude se caractérise par un climat aride soumis aux influences désertiques provenant du Sud-Ouest, et celles méditerranéennes relativement doux à l'Est et au Nord. Le régime de pluie se caractérise par une forte irrégularité annuelle, une extrême variabilité saisonnière et une récurrence de la sécheresse. 
Tableau 1

Paramètres de pluie dans quelques stations de la zone d'étude pour la période 1960-2000

\begin{tabular}{llccc}
\hline \multicolumn{1}{c}{ Station } & \multicolumn{1}{c}{ Localisation } & Pm & Pmax & Pmin \\
\hline Toujène & Jebel & 233,2 & 649,8 & 89,0 \\
Béni Khédache & Jebel & 231,8 & - & - \\
Ksar Hallouf & Vallée de montagne & 168,5 & 394,5 & 45,5 \\
Ksar Jedid & Piémont & 159,8 & 357,0 & 7,5 \\
Alamet Mechlouch & Plaine & 145,6 & 364,0 & 6,0 \\
\hline
\end{tabular}

Pm : Précipitation moyenne annuelle ; Pmax : Précipitation maximale ;

Pmin : Précipitation minimale

Source : Ferchichi 1996

Les précipitations moyennes interannuelles sont comprises entre $140 \mathrm{~mm}$ et $240 \mathrm{~mm}$ (Floret et Pontanier 1982, Ferchichi 1996) avec des différences sensibles entre les différents milieux. En effet, les montagnes s'échappent en partie de ce régime ordinaire grâce à l'influence de l'altitude et bénéficie de quantités de pluie supérieures $200 \mathrm{~mm}$ (Tab. 1). L'irrégularité du climat est telle que la région peut connaitre des années pluvieuses pendant lesquelles la quantité de pluie peut dépasser la moyenne annuelle en quelques jours (Abichou 2009). En revanche, La sécheresse peut s'installer durant une à trois années durant lesquelles les quantités annuelles de pluie descendent à moins de $50 \mathrm{~mm}$.

Les températures moyennes annuelles sont aux alentours de $19,5^{\circ} \mathrm{C}$. Elles sont aussi variables entre les différents secteurs (Tab. 2). Ce paramètre $\mathrm{du}$ climat présente une influence capitale sur le comportement des organismes par le contrôle qu'il exerce sur l'ensemble des processus vitaux des espèces végétales. La croissance, l'activité végétative, la reproduction sont étroitement dépendantes de la température.

Tableau 2

Caractéristiques de la température dans quelques stations du Sud-Est

\begin{tabular}{lccc}
\hline Station & Moyenne & $\mathbf{M}\left({ }^{\circ} \mathbf{C}\right)$ & $\mathbf{m}\left({ }^{\circ} \mathbf{C}\right)$ \\
\hline Matmata & 18,9 & 35,2 & 5,5 \\
Médenine & 20,5 & 36,8 & 6,6 \\
\hline
\end{tabular}

$\mathbf{M}$ : Moyenne des maxima du mois le plus chaud ; $\mathbf{m}$ : Moyenne des minima du mois le plus froid

Source : Hanafi 2008 
L'ensemble de ces caractéristiques permettent de classer la zone d'étude pour l'ensemble des bioclimats délimités par Emberger (1955) et Le Houérou (1959), dans l'étage bioclimatique méditerranéen aride avec deux sous-étages: L'aride supérieur à hivers tempérés caractérisant les montagnes (Toujène, Zmerten, Ksar Hallouf, Béni Khédache, Zammour) et l'aride inférieur à hivers doux caractérisant les piémonts et les plaines (Ksar Jedid, Bhayra, Halg Jmal, Chouamakh, Dkhilet Toujène) (Floret et al 1978, Floret et Pontanier 1982, Ferchichi 1996). Le facteur topographique est ici fondamental pour expliquer ces différences entre ces zones. En effet, les monts de Matmata permettent une meilleure douceur des températures ainsi qu'une meilleure exposition aux influences fraiches et humides provenant de la Mer Méditerranée distante d'une trentaine de kilomètre. C'est cette condition qui va expliquer par la suite la présence d'un matorral méditerranéen dans cette région. Mais quoi qu'il en soit de ces conditions topographiques, le bioclimat aride peut présenter pour certaines années sèches des tendances proches de l'étage saharien (le cas des années 1999-2002), et pour d'autres plus pluvieuses, les caractères des étages semi-arides et même subhumides (le cas de l'année 2003-2004 ou 2017-2018).

La faiblesse des précipitations dans la zone d'étude explique l'indigence du réseau hydrographique qui est assez complexe et basé sur une forte complémentarité entre les eaux de surface et les aquifères souterrains (Romagny et al 2003). Les eaux de surface s'accumulent dans un réseau hydrographique relativement dense qui ne fonctionne qu'à la suite de très fortes pluies. Les cours d'eau peuvent rester deux à trois années sans écoulement (Hanafi 2008). Quant aux eaux souterraines de la région, elles sont importantes en présence des nappes phréatiques et de celles profondes avec un écoulement général vers le NE. Selon Romagny et al (2003), les nappes phréatiques s'étalent sur la partie montagneuse au niveau des localités de Toujène, Dkhilet Toujène et El Bhayra et sont alimentées essentiellement par les eaux de surface.

Pour les nappes profondes, trois ont été identifiées; la nappe jurassique de Béni Khédache, qui présente une zone d'alimentation préférentielle à l'ouest; la nappe du Grès de Trias au niveau de Ksar Jedid qui est limitée au nord par les affleurements du mont Tebaga et enfin la nappe jurassique et crétacé à l'Est de Dkhilet Toujène, qui assure 
l'alimentation en eau potable des principales villes du gouvernorat de Médenine (Tab. 3), (Ouessar et Yahyaoui 2006).

Tableau 3

Caractéristiques des différentes nappes profondes dans la région

\begin{tabular}{lccc}
\hline \multicolumn{1}{c}{ Nappes } & $\begin{array}{c}\text { Profondeur de } \\
\text { captage }(\mathbf{m})\end{array}$ & $\begin{array}{c}\text { Salinité } \\
(\mathbf{g} / \mathbf{l})\end{array}$ & $\begin{array}{c}\text { Débit } \\
\mathbf{( 1 / s )}\end{array}$ \\
\hline - Jurassique de Béni Khédache & $200-300$ & $1.7-6$ & 16 \\
- Grés de Trias au niveau de Ksar & $100-300$ & $1.0-3$ & 90 \\
Jedid & & & \\
- Jurassique et Crétacé de Dkhilet & $100-300$ & $1.5-5$ & 350 \\
Toujène & & & \\
\hline
\end{tabular}

Source : Ouessar et Yahyaoui 2006

\section{Une population bien ancrée dans son territoire}

La zone d'étude a, depuis l'antiquité, été le théâtre de mouvements humains et de dynamiques complexes d'occupation spatiale, qui portent la signature des hommes sur leur environnent et leur capacité d'adaptation à des milieux contraignants. D'après Guillaume et al (2003), la région se caractérise par une occupation ancienne qui remonte à la fin de la période Romaine. Par ailleurs, et à l'image de tout le Sud Tunisien, elle a connu une série de mutations qui a touché les structures économiques et sociales et par conséquent, les modes d'exploitation et d'occupation du sol (Abaab 1986, Guillaume et al 2003). Ces transformations sont caractérisées par le passage d'une occupation et d'une utilisation traditionnelle de l'espace et de ses ressources, dans le cadre d'un mode de vie nomade et semi-nomade, à une occupation et une utilisation relativement intensives dans le cadre d'un mode de vie sédentaire, basé sur une diversification des activités économiques.

Selon Guillaume et al. (2003), la zone d'étude est aujourd'hui occupée par une population berbéro-arabe installée dans la plupart des cas dans des villages en montagne. Au niveau de la partie du gouvernorat de Médenine, cette population est marquée par une mosaïque d'habitants appartenant à l'ancienne confédération des Ouerghemma. Cette population a depuis longtemps été caractérisée par une emprise rationnelle sur 
l'espace avec une occupation traditionnelle des terres à travers un mode de vie sédentaire en montagne, et nomade à semi-nomade en piémonts et en plaines. L'utilisation des terres et des ressources a été basée sur les activités pastorales bien adaptée aux contraintes du milieu aride (Abaab 1986). Les habitants des montagnes ont pratiqué l'élevage (ovin, caprin, camelin) et ont aussi développé l'arboriculture (olivier, figuiers, amandiers, etc.), en plus de quelques cultures céréalières fondées sur les eaux de ruissellement des jessour ${ }^{4}$. Cette utilisation a donné naissance à un système de production agro-pastoral extensif associé à des formes de semi-nomadisme (Guillaume et al 2003). A partir des années 1960, la population a connu une importante croissance de ses effectifs et la région a bénéficié de quelques projets de développement agricole entrepris essentiellement par l'Etat. Ceci a été l'origine d'une déstabilisation écosystèmes et des communautés paysannes et d'une grande emprise sur le milieu manifestée par une expansion des plantations d'arbres notamment au niveau des interfluves encroûtés (Hanafi 2008).

\section{Matériel et méthodes}

Afin d'étudier les différentes unités végétales, une base de données sous SIG a été créée afin de dresser la carte des groupements végétaux. Cette cartographie a été nécessaire pour déterminer les conditions actuelles de la flore (composition floristique, état de dégradation, type d'exploitation), pour identifier les diverses unités de la végétation ainsi que pour analyser les données sur la diversité spécifique et écosystémique dans cette région. Cette cartographie est passée par trois principales étapes.

La première étape a consisté à une réalisation d'une photointerprétation visuelle des différentes unités de milieu. Ceci a été possible grâce à l'usage d'une image satellitale extraite de Google Earth

\footnotetext{
${ }^{4}$ Les jessour constituent des d'ouvrages de petite hydraulique traditionnelle (élévations en terres dressées dans les lits d'oueds), conçus dans les montagnes du Sud Tunisien pour retenir l'excédent en eau et en sol venant des impluviums et leur exploitation pour l'agriculture. Le Jesser (singulier) se compose de l'impluvium, du lit de l'oued et de l'aménagement dans le ravin pour barrer l'écoulement, appelé aussi localement tabia ou katra (Bonvallot 1986, Ben Ouezdou 2001).
} 
de 2016. Cette image a été accompagnée par la carte topographique de Gabès au 1/200.000. La délimitation visuelle des unités homogènes à partir de Google Earth a permis de dégager 85 polygones. Ces derniers ont été exportés sur ArcView afin de créer une base de données géo-spatiale et préparer la visite du terrain. Le plan d'échantillonnage a été choisi afin d'assurer un passage systématique sur toute la zone d'étude. La deuxième étape, a consisté à la réalisation d'une mission de terrain afin de valider le découpage des unités obtenues par la photointerprétation et pour réaliser 62 relevés de végétation et du milieu de type Braun-Blanquet (1932). Pour chaque site visité, une liste d'espèces végétales a été déterminée. Par ailleurs, un coefficient d'abondancedominance a été attribué à chaque espèce et qui traduit son importance relative par rapport aux autres espèces. Parallèlement à l'inventaire de la végétation, une observation des caractères géographiques du site a été effectuée (topographie, géologie, géomorphologie, formations superficielles, occupation humaine) ce qui a permis de mieux comprendre les résultats du suivi.

La dernière étape a consisté à la réalisation d'une Analyse Factorielle des Correspondances (AFC) suivie d'une Classification Ascendante Hiérarchique pour l'ensemble des relevés effectuées (Benzecri 1964, Bry 1996, Escofier et Pages 2002). L'objectif de ce traitement étant d'identifier les associations, les groupements et les faciès de végétation à partir des données phytoécologiques recueillies sur le terrain. Ce travail a ainsi permis d'identifier les différents groupements végétaux reconnus dans la zone étudiée et a servi comme base pour l'analyse de la diversité floristique.

\section{Résultats et discussion}

\section{Analyse multivariée des données écologiques}

Une première analyse a été effectuée sur les 62 relevés réalisés mais n'a pas été retenue. En effet, quatre relevés ont fortement tiré le nuage de point dans la représentation graphique ce qui a rendu difficile l'interprétation des axes factoriels. L'examen de ces relevés a montré qu'ils englobent des espèces qui n'ont été rencontrées qu'une ou deux fois dans le milieu. C'est le cas d'Olea europaea, Pistacia lentiscus, Ononis natrix ssp. falcata, Lycium arabicum, Ephedra altissima. D'autres espèces 
rencontrées ont des caractéristiques écologiques différentes de celles des milieux dans lesquels elles ont été rencontrées (Ex: Umbilicus sp). Une deuxième AFC a, alors, été effectuée sur les 58 relevés restants, totalisant 82 espèces. Le tableau 4 relatif aux valeurs propres et aux pourcentages d'inertie montre une bonne structuration du nuage des points notamment sur les trois premiers axes retenus alors pour l'analyse.

Tableau 4

Valeurs propres et pourcentages d'inertie des quatre premiers axes de l'AFC

\begin{tabular}{lcccc}
\hline Axe & F1 & F2 & F3 & F4 \\
\hline Valeur propre & 0,393 & 0,274 & 0,273 & 0,244 \\
Pourcentage d'Inertie (\%) & 10,316 & 7,203 & 7,158 & 6,399 \\
\hline
\end{tabular}

\subsection{Description et signification écologique de l'axe 1}

Sur le premier axe se présentent les espèces suivantes avec un poids important ${ }^{5}$ :

\begin{tabular}{cl}
\hline Côté négatif de l'axe & \multicolumn{1}{c}{ Côté positif de l'axe } \\
\hline & - Rosmarinus officinalis var. troglodytarum (106) \\
& - Thymus capitatus (89) \\
Absence d'espèces ayants & - Genista microcephala var. tripolitana (81) \\
un poids important & - Scilla sp (72) \\
& - Thymus hirtus var. algeriensis (54) \\
& - Hypericum sp (53) \\
& - Juniperus phoenicea (53) \\
\hline
\end{tabular}

${ }^{5}$ Il s'agit d'un poids relatif à chaque espèce végétale déterminé par l'analyse factorielle en fonction de l'importance relative des espèces dans leur milieu. Ainsi les espèces ayant le plus de poids sont généralement déterminantes dans le façonnement des groupements et indiquent souvent un lien fort avec les facteurs mésologiques (Climat, sol, topographie, action humaine...). 


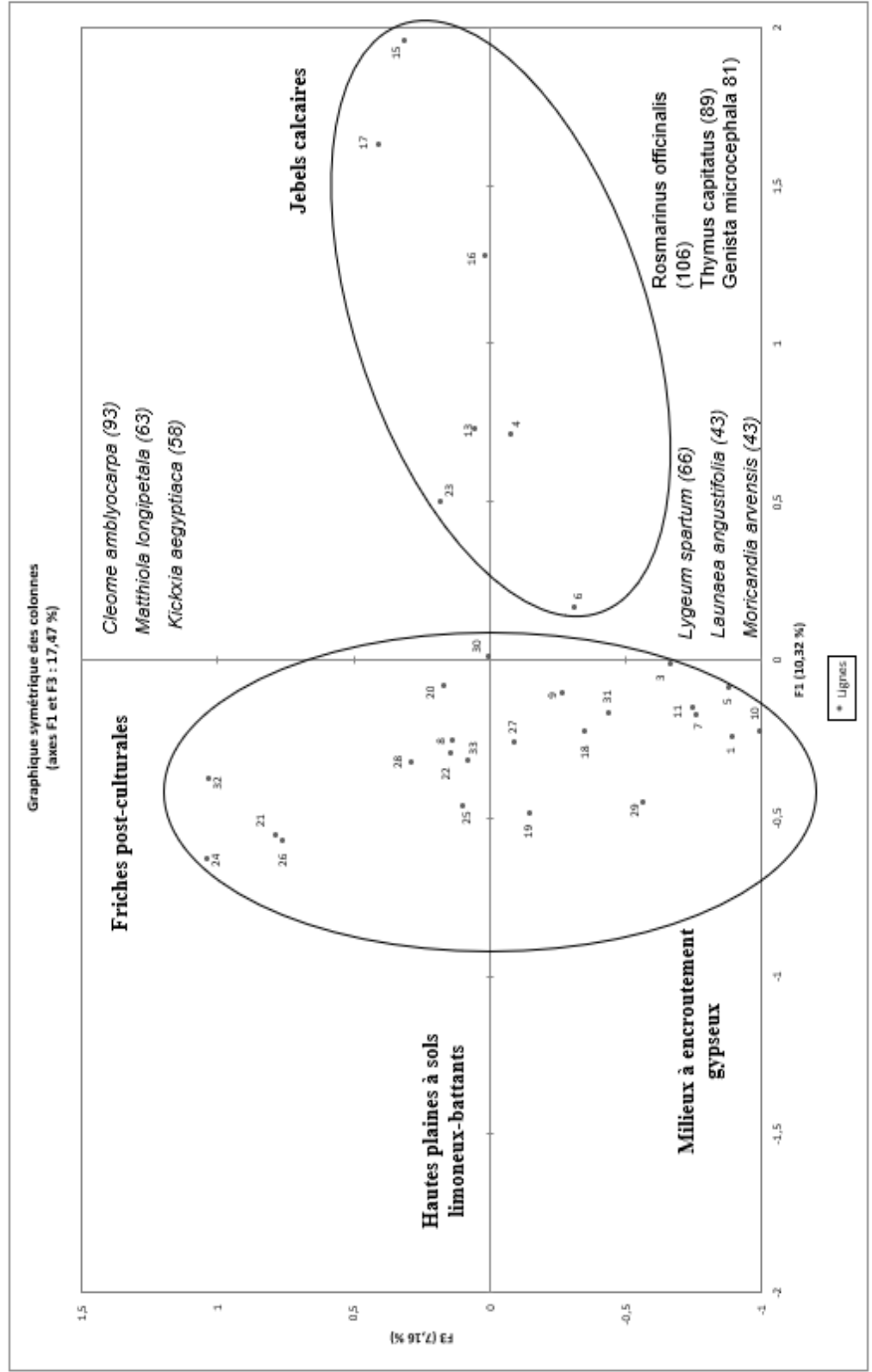

Fig. 2. Signification écologique des axes 1-2 de 1'AFC appliquée aux données présence-absence de la végétation de la zone d'étude 


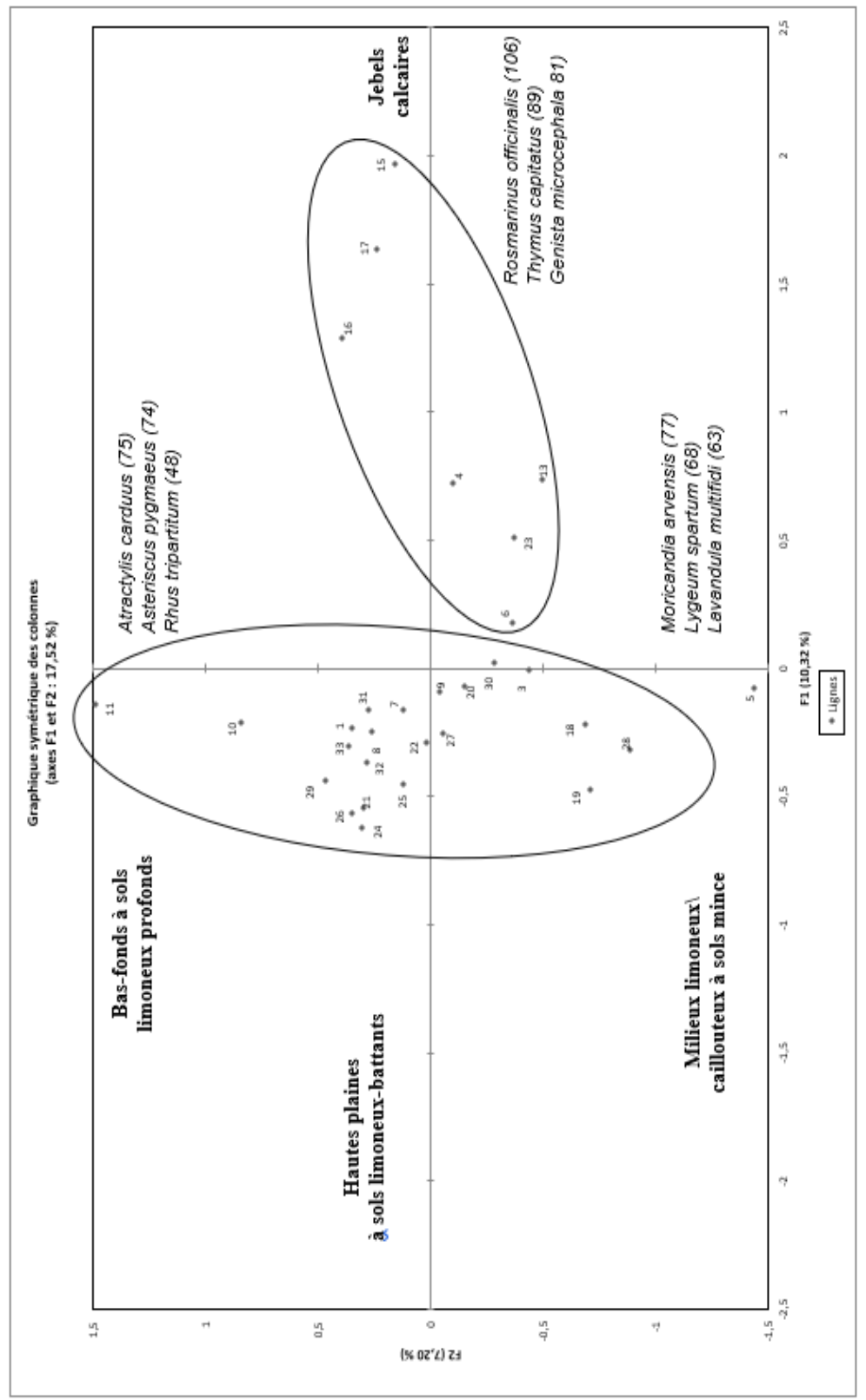

Fig. 3. Signification écologique des axes 1-3 de l'AFC appliquée aux données présence-absence de la végétation de la zone d'étude. 
Les espèces présentes sur le côté négatif de ce premier axe ont un poids très faible ce qui rend difficile l'interprétation de l'axe. Sur le côté positif, les espèces qui ont le plus de poids sont des espèces méditerranéo-steppiques (Thymus capitatus, Thymus hirtus var. algeriensis, Luniperus phoenicea) en mélange avec des espèces préforestières (Rosmarinus officinalis var. troglodytarum). Cet axe présente ainsi, une seule partie claire avec une végétation préforestière indiquant les montagnes calcaires aux sols rocailleux de l'étage aride supérieur.

\subsection{Description et signification écologique de l'axe 2}

L'analyse du deuxième axe montre que plusieurs espèces marquent ses deux pôles positif et négatif :

\begin{tabular}{ll}
\hline \multicolumn{1}{c}{ Côté négatif de l'axe } & Côté positif de l'axe \\
\hline - Atractylis flava (75) & - Moricandia arvensis (77) \\
- Asteriscus pygmaeus (74) & - Lygeum spartum (68) \\
- Lycium sp. (57) & - Lavandula multifida (63) \\
- Rhus tripartitum (48) & \\
- Stipa tenacissima (46) & \\
\hline
\end{tabular}

Les espèces marquant le côté négatif de l'axe se développent sur les sols calcaires grossiers, sur les abrupts rocheux (Rhus tripartitum, Stipa tenacissima, Lycium sp) et sur les sols limoneux (Asteriscus pygmaeus, Atractylis flava). Sur le plan bioclimatique, ces espèces indiquent généralement l'aride inférieur. Sur le côté positif de cet axe se concentrent des espèces méditerranéo-steppiques (Lavandula multifida) avec des espèces gypsophiles des piémonts limoneux (Lygeum spartum, Moricandia arvensis). Cet axe indique un passage du bioclimat aride supérieur à aride inférieur et semble en relation avec la texture du sol. Il représente un gradient décroissant des falaises et des piémonts aux sols limoneux et rocailleux vers les piémonts aux sols limoneux-gypseux (Fig. 2). 
LA VÉGÉTATION MÉDITERRANÉENNE ARIDE DANS LES AIRES DE TRANSITION : TYPOLOGIE, RICHESSE ET DÉFIS DE VALORISATION. CAS DES MONTS DE MATMATA (SUD TUNISIEN)

\subsection{Description et signification écologique de l'axe 3}

Le troisième axe de l'analyse oppose les espèces suivantes:

\begin{tabular}{ll}
\hline \multicolumn{1}{c}{ Côté négatif de l'axe } & Côté positif de l'axe \\
\hline - Lygeum spartum (66) & - Cleome amblyocarpa (93) \\
- Launaea angustifolia (43) & - Matthiola longipetala (63) \\
- Moricandia arvensis (43) & - Kickxia aegyptiaca (58) \\
& - Calendula arvensis (44) \\
\hline
\end{tabular}

Les espèces ayant le plus de poids sur le côté négatif de l'axe 3 sont des espèces gypsophiles caractérisant les glacis d'érosion à croûte gypseuses et indiquant l'étage bioclimatique aride inférieur (Launaea sp, Lygeum spartum, Moricandia arvensis). Le côté positif de l'axe est représenté par des espèces de l'aride inférieur qui se développent sur les substrats sablo-limoneux fins et poussent au niveau des friches postculturales (Matthiola longipetala, Cleome amblyocarpa). Les relevés de cet axe sont localisés en aval de la zone d'étude et représentent un gradient écologique allant des plaines à encroûtements gypseux vers les friches post-culturales (Fig. 3).

\section{Des groupements à dominante édaphique et climatique}

\subsection{Typologie de la végétation de Matmata}

La Classification Ascendante Hiérarchique appliquée aux données présence/absence a distribué les relevés de végétation sur deux ensembles A et B. Chacun de ces ensembles a été subdivisé en plusieurs sous-ensembles et groupes renfermant chacun un ou plusieurs groupements de végétation (Fig. 4). La réalisation d'une AFC appliquée sur les données abondance-dominance de chacune de ces groupes phytoécologiques a permis l'identification des groupements présents.

\subsubsection{Groupements végétaux de l'ensemble A}

L'ensemble A se subdivise en deux sous-ensembles : 


\section{- Le sous ensemble $\mathrm{A} 1$ :}

Groupe A11 : il est représenté par deux sous-groupes :

+ Sous-groupe A111: il rassemble les relevés indiquant les montagnes, les piémonts et les plaines des milieux aux sols squelettiques à croûte et encroûtement calcaire et à sol limoneux mince avec des types de végétation indiquant l'association à Seriphidium herba-alba et Haloxylon scoparium (Le Houérou 1959) à savoir :

- Faciès type,

- Sous -association à Gymnocarpos decander (Le Houérou 1959),

- Faciès à Stipa tenacissima (Le Houérou 1959),

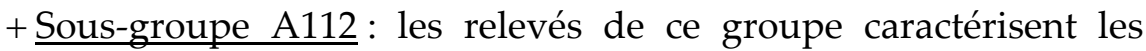
milieux sableux et gypseux de l'association à Rhanterium suaveolens et Artemisia campestris

- Faciès type,

- Sous-association à Retama raetam (Le Houérou 1959),

- Sous association à Lygeum spartum (Le Houérou 1959),

- Faciès à Calycotome villosa et Astragalus armatus ssp. tragacanthoides (Hanafi 2008),

Groupe A12 : il rassemble des relevés indiquant les collines calcaires et est représenté par l'association à Seriphidium herba-alba et Haloxylon scoparium (Le Houérou 1959) :

- Faciès à Stipa tenacissima (Le Houérou 1959).

- Le sous ensemble A2 : il représente des groupements caractérisant les épandages et les lits d'oued avec :

$\checkmark$ Association à Artemisia campestris et Thymus capitatus (Le Floc'h 1973)

- Faciès type,

$\checkmark$ Association à Deverra tortuosa et Haplophyllum vermiculare (Le Houérou 1959)

- Faciès type, 


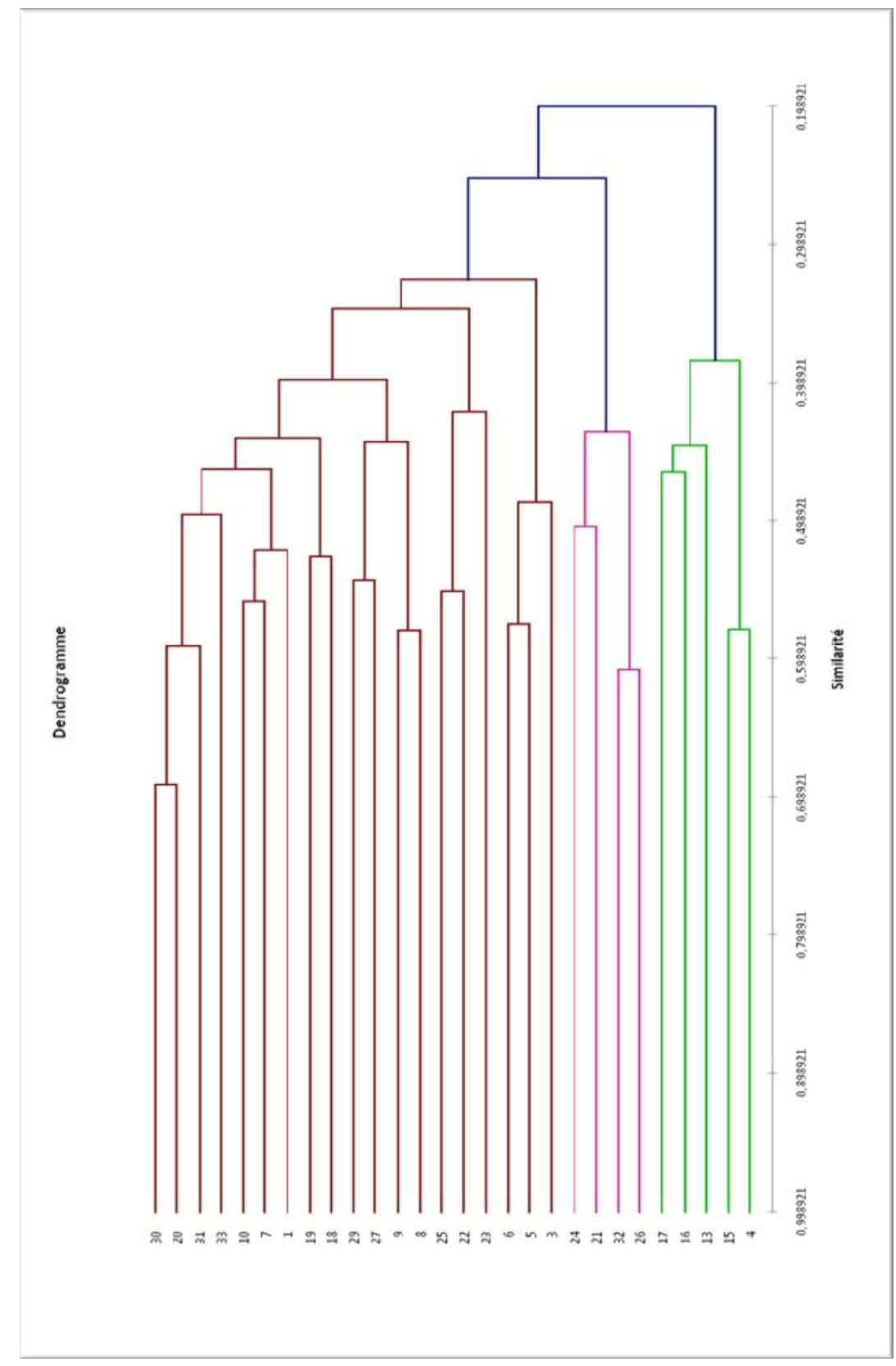

Fig. 4. CAH des données présence/absence de la végétation de la zone d'étude

Source: Aridhi 2016. 


\subsubsection{Groupements végétaux de l'ensemble $B$}

Selon la CAH le groupe B est subdivisé en 2 sous ensembles :

- Le sous ensemble B1: il rassemble des relevés indiquant les fronts des cuestas et les versants exposés au froid hivernal de l'étage bioclimatique méditerranéen aride supérieur. Il se caractérise par l'association à Genista microcephala var. tripolitana et Teucrium alopecurus

- Faciès type,

- Le sous ensemble B2 : il caractérise les revers des montagnes et est représenté par les associations suivantes :

$\checkmark$ Association à Genista microcephala var. tripolitana et Teucrium alopecurus (Le Houérou 1959) :

- Faciès à Juniperus phoenicea et Rosmarinus officinalis var. troglodytarum (Le Houérou 1959),

$\checkmark$ Association à Seriphidium herba-alba et Haloxylon scoparium (Le Houérou 1959) :

- Faciès à Stipa tenacissima (Le Houérou 1959).

\subsection{Biologie et écologie des unités végétales}

La biologie et l'écologie des différents groupements identifiés sont en grande partie expliquées par des facteurs physiques (topographie, sols, climat, réseau hydrographique, etc.). Avec ces facteurs, l'action humaine très ancienne dans la région, joue un rôle important dans le façonnement du paysage végétal. Ces différents facteurs identifiés dans la zone d'étude ont permis de mettre en évidence neuf unités de végétation classées selon quatre types de milieu morpho-pédologiques (Fig. 5).

\subsubsection{Les unités végétales des montagnes}

Ces unités s'étendent sur environ $24,3 \%$ de la zone d'étude. Trois types se distinguent en fonction du climat et du type de sol.

- Matorral bas à Genévrier de Phénicie (Juniperus phoenicea) et Romarin (Rosmarinus officinalis var. troglodytarum) : C'est un matorral méditerranéen qui constitue un groupement de dégradation de l'association à Pinus halepensis et Helianthemum ellipticum 
(Le Houérou 1959, 1969). Il se rencontre au niveau des sommets de Matmata (Baten El Khadhra et Menzela...) avec une végétation à base de Juniperus phoenicea et Rosmarinus officinalis var. troglodytarum. Le matorral trouvé appartient à l'étage méditerranéen aride supérieur et présente quelques espèces endémiques. C'est le cas Thymus hirtus var. algeriensis (endémique Nord-Africaine) et de Rosmarinus officinalis var. troglodytarum (endémique de Matmata). Le cortège floristique qui caractérise ce matorral est riche en espèces méditerranéennes (Juniperus phoenicea, Rosmarinus officinalis var. troglodytarum, Stipa tenacissima, Genista microcephala var. tripolitana, Thymus hirtus var. algeriensis, Rhus tripartitum, Ruta chalepensis var. bracteosa, Calycotome villosa var. intermedia). Toutefois, ce matorral ne s'étend que sur seulement 6,1\% des terres (Tab. 5, Fig. 5) au niveau des revers de la Cuesta aux alentours des villages de Toujène et de Zmerten sur des sols squelettiques et des croûtes calcaires. Il est aujourd'hui sensible à la dégradation d'autant plus qu'il est de plus en plus réduit quantitativement et qualitativement (Hanafi 2008). C'est un type de végétation assez original dans le Sud-Est tunisien puisqu'il n'existe que dans cette partie de Matmata (Hanafi 2008).

- Matorral bas à Genêt (Genista microcephala var. tripolitana), Thym d'Algérie (Thymus hirtus var. algeriensis) et Romarin (Rosmarinus officinalis var. troglodytarum): Ce matorral représente un type de dégradation de l'association à Genista microcephala var. tripolitana et Teucrium alopecurus et appartient à l'étage méditerranéen aride supérieur. Il est dominé par à Genista microcephala var. tripolitana, Thymus algeriensis et Rosmarinus officinalis var. troglodytarum, Stipa retorta et Diplotaxis harra. Par rapport à l'unité précédente, ce matorral se caractérise par la disparition de quelques espèces méditerranéennes notamment Juniperus phoenicea. Seul Rosmarinus officinalis var. troglodytarum persiste encore et parfois avec des grandes touffes. Le cortège floristique de ce matorral est aussi riche et se développe sur quelques revers de montagnes sur des sols limoneux. Il est dominé par des espèces essentiellement steppiques le cas d'Atractylis serratuloides, Echinops spinosus, Diplotaxis harra, Seriphidium herba-alba, etc. Ce matorral s'étend sur environ $17,7 \%$ 
de la zone d'étude et est marqué essentiellement par la forte présence de l'activité humaine (cultures derrières les jessour; pâturages ; coupe d'alfa, du thym et du romarin...) ce qui menace les espèces rares par la disparition.

- Pseudo-steppe à Astragale (Astragalus armatus ssp. tragacanthoides) et Calycotome (Calycotome villosa var. intermedia) : Au niveau de la route entre Béni Khédache et Ksar Hallouf s'étend une steppe assez originale de la zone, dominée essentiellement par Astragalus armatus ssp. tragacanthoides et Calycotome villosa var. intermedia. Elle représente un faciès de l'association à Rhanterium suaveolens et Artemisia campestris. Son originalité est due à cette combinaison non rencontrée auparavant dans la région aride tunisienne entre Rhanterium suaveolens, espèce typiquement steppique et Calycotome villosa var. intermedia qui est rattachée au cortège floristique du matorral méditerranéen (Hanafi 2008). Le recouvrement total des pérennes est important dépassant $15 \%$ et le cortège floristique est riche renfermant notamment Diplotaxis harra, Plantago albicans, Helianthemum kahiricum, Polygonum equisetiforme, Atractylis serratuloides, Deverra tortuosa et Artemisia campestris. Cette steppe s'étend sur une vaste poche intramontagnarde remplie de limons et de sable. Elle est limitée dans l'espace et ne représente que 0,5\% de la superficie totale de la zone d'étude (Fig. 5, Tab. 5).

Tableau 5

Répartition (\%) de la végétation en fonction des différents types de milieu de zone d'étude

\begin{tabular}{lcc}
\hline \multicolumn{1}{c}{ Unité } & $\begin{array}{c}\text { Superficie } \\
\text { en ha }\end{array}$ & $\%$ \\
\hline $\begin{array}{l}\text { Montagnes } \\
\text { Piémonts et collines calcaires } \\
\text { encroûtés }\end{array}$ & 13103 & 24,3 \\
$\begin{array}{l}\text { Plaines sablo-limoneuses et } \\
\text { gypseuses }\end{array}$ & 22823 & 42,3 \\
$\begin{array}{l}\text { Épandages et fonds d'oueds } \\
\text { Habitat et culture }\end{array}$ & 2231 & 4,1 \\
\multicolumn{1}{c}{ Total } & 1556 & 2,9 \\
\hline
\end{tabular}

Source : Données de terrain 


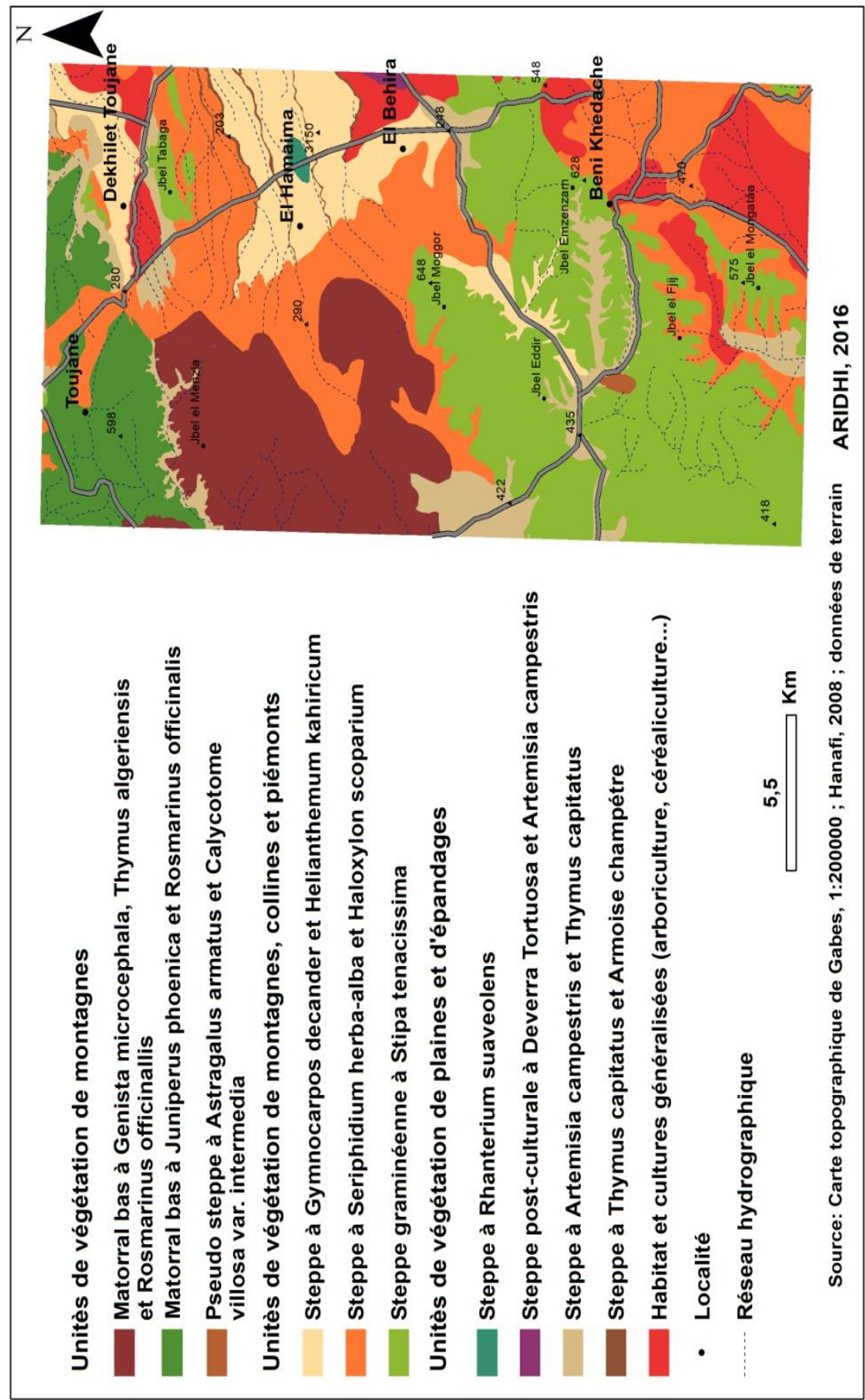

Fig. 5. Carte de la végétation actuelle de la zone d'étude 
Tableau 5

Répartition (\%) de la végétation en fonction des différents types de milieu de zone d'étude

\begin{tabular}{lcc}
\hline \multicolumn{1}{c}{ Unité } & $\begin{array}{c}\text { Superficie } \\
\text { en ha }\end{array}$ & $\%$ \\
\hline $\begin{array}{l}\text { Montagnes } \\
\text { Piémonts et collines calcaires } \\
\text { encroûtés }\end{array}$ & 13103 & 24,3 \\
$\begin{array}{l}\text { Plaines sablo-limoneuses et } \\
\text { gypseuses }\end{array}$ & 22823 & 42,3 \\
$\begin{array}{l}\text { Épandages et fonds d'oueds } \\
\text { Habitat et culture }\end{array}$ & 2231 & 4,1 \\
\multicolumn{1}{c}{ Total } & 1556 & 2,9 \\
\multicolumn{1}{c}{14287} & 26,5 \\
\hline
\end{tabular}

Source : Données de terrain

2.2.2. Les unités végétales des collines et des piémonts

Ces unités représentent $42,3 \%$ de la superficie de la zone d'étude et caractérisent les collines et les piémonts. Elles se répartissent en trois types distincts :

- Steppe à Armoise blanche (Seriphidium herba-alba) et Remth (Haloxylon scoparium): Cette steppe est représentée par le faciès type de l'association à Seriphidium herba-alba et Haloxylon scoparium résultant de la dégradation de la steppe graminéenne à Stipa tenacissima. Elle est dominée essentiellement par Seriphidium herbaalba, Haloxylon scoparium et Helianthemum kahiricum. Le cortège floristique de cette steppe est riche avec la présence d'espèces steppiques notamment Astragalus armatus ssp. tragacanthoides, Stipa tenacissima, Gymnocarpos decander, Asparagus albus, Herniaria fontanesii, Scorzonera undulata, Launaea angustifolia, Teucrium polium ainsi que des espèces saharo-arabiques comme Asteriscus pygmaeus, Hippocrepis areolata. Cette steppe représente environ $16,8 \%$ de la zone d'étude et se développe sur les sols limono-sableux à croûtes et encroûtements calcaires. Elle se localise essentiellement sur les piémonts des montagnes et les collines limoneuses aux alentours de Béni Khédache et Halg Jmel ainsi que sur quelques parties des plaines au niveau de Dkhilet Toujène où Stipa tenacissima devienne rare. 
- Steppe graminéenne à Alfa (Stipa tenacissima) : elle s'étend sur un espace relativement important représentant $16,2 \%$ de la superficie de la zone d'étude. Elle fait partie de l'association à Seriphidium herba-alba et Haloxylon scoparium et présente une transition entre l'étage bioclimatique méditerranéen supérieur et celui inférieur. Malgré cette transition bioclimatique, cette unité est marquée par l'absence d'espèces méditerranéennes surtout Rosmarinus officinalis var. troglodytarum. C'est une steppe pauvre de point de vue floristique, dominée surtout par Stipa tenacissima, et par des espèces souvent en état de dégradation notamment Helianthemum kahiricum, Diplotaxis harra, Seriphidium herba-alba. Ce type de végétation se localise essentiellement sur les fronts ensoleillés de la cuesta au sud de la zone d'étude au niveau de Béni Khédache et domine aussi sur quelques collines aux alentours de Dkhilet Toujène. L'usage collectif des ressources de cette steppe est à l'origine de sa dégradation notamment quantitative (recul du nombre de pieds de Stipa tenacissima) (Hanafi 2008).

- Steppe à Khachra (Gymnocarpos decander) et Kourchid (Helianthemum kahiricum) : Cette steppe est représentée par la sous-association à Gymnocarpos decander de l'association à Seriphidium herba-alba et Haloxylon scoparium. Elle est dominée par Gymnocarpos decander, Helianthemum kahiricum, Atractylis serratuloides et Astragalus armatus ssp. tragacanthoides. Le cortège floristique est généralement pauvre avec la présence de Scorzonera undulata, Kickxia aegyptiaca, Asteriscus pygmaeus, Herniaria fontanesii et Stipa retorta. Le recouvrement total des espèces pérennes est en général faible aux alentours de $10 \%$. Cette steppe s'étend sur environ 9,3\% de la superficie totale de la zone d'étude et se localise sur un ensemble de petites collines et sur une partie de la plaine dans la partie centrale de la zone au niveau de Ksar Jedid. Elle se développe sur des sols à croûte calcaire superficielle surmontée d'un faible horizon alluvionnaire de texture sablo-limoneuse (Le Houérou 1969). Certaines terres de cette steppe sont souvent cultivées par les céréales mais suite à la pression anthropique croissante sur ces ressources, cette culture est 
remplacée par les plantations arboricoles malgré la faible productivité des terres (Hanafi 2008).

\subsubsection{Les unités végétales des plaines}

Ces unités, et $d u$ fait de la faible étendue des plaines dans cette zone d'étude, ne s'étendent que sur $4,1 \%$ de la superficie de la zone d'étude (Tab. 5). Elles sont représentées par deux steppes :

- Steppe à Arfej (Rhanterium suaveolens) et Armoise champêtre (Artemisia campestris) : elle est représentée par le faciès typique de l'association à Rhanterium suaveolens et Artemisia campestris. Elle est dominée par Rhanterium suaveolens, Artemisia campestris et Helianthemum lippi var. sessiliflorum. Le cortège floristique est riche en espèces saharo-arabiques notamment Gymnocarpos decander, Matthiola longipetala, Helianthemum kahiricum, ainsi que des espèces méditerranéennes notamment Allium roseum, Astragalus caprinus, Launaea resedifolia, Scorzonera undulata et Artemisia campestris. Cette steppe est étendue sur seulement $2 \%$ de la superficie de la zone d'étude au niveau de la plaine. Elle colonise les terrains aux sols limono-sableux profonds parfois à nodules calcaires (Le Floc'h 1973, Hanafi 2008).

- Steppe à Armoise champêtre (Artemisia campestris) et Gouzzah (Deverra tortuosa) : cette steppe post-culturale est composée de trois groupes différents. Il s'agit de la sous-association à Retama raetam de l'association à Rhanterium suaveolens et Artemisia campestris, l'association à Deverra tortuosa et Haplophyllum vermiculare et sa sous-association à Atractylis serratuloides, Lygeum spartum, Retama raetam (Boukhris et Lossaint 1972, Le Houérou 1959). Elle est dominée essentiellement par Deverra tortuosa, Retama raetam et Artemisia campestris. Le cortège floristique est riche. Il se caractérise essentiellement par des espèces saharo-arabiques notamment Salvia aegyptiaca, Helianthemum lippii var. sessiliflorum, Cleome amblyocarpa, Diplotaxis harra et Matthiola longipetala. Cette steppe ne présente que 2,1\% de la zone d'étude. Elle est rencontrée des sols limono-sableux parfois encroûtés par le calcaire. Sa faible étendue est notamment expliquée par la mise en place sur cette plaine d'une culture arboricole (Hanafi 2008). 


\subsubsection{Les unités végétales d'épandages et de fonds d'oueds}

Les épandages et les fonds d'oueds ne sont représentés dans la zone d'étude que par une seule unité de végétation. Il s'agit de la steppe à Artemisia campestris et Thymus capitatus. Cette steppe est représentée par le faciès type de l'association à Artemisia campestris et Thymus capitatus (Le Floc'h 1973) et représente les fonds d'oueds et les bas-fonds caillouteux de la zone d'étude. Elle est dominée par Artemisia campestris, Echinops spinosus, Enarthrocarpus clavatus et Deverra tortuosa. Le recouvrement total de la végétation pérenne est en général supérieur à $10 \%$ et présente une steppe riche en espèces avec notamment Salvia aegyptiaca, Kickxia aegyptiaca, Matthiola longipetala, Seriphidium herba-alba, Ziziphus lotus, Calycotome villosa var. intermedia, Launaea residifolia, Polygonum equisetiforme et Cleome amblyocarpa. Cette steppe domine tous les fonds d'oueds et les épandages dans la zone d'étude et s'étend sur environ $2,9 \%$ de la superficie totale. Selon Hanafi (2008), les parcours de cette steppe sont aujourd'hui aménagés en terrasses de culture et protégés contre l'écoulement par des tabias.

\section{La diversité végétale dans les monts de Matmata}

La diversité végétale a pris une place de plus en plus importante parmi les grandes questions environnementales comme le changement climatique ou la perturbation de la couche d'ozone (Lévèque 2001). L'étude de cette question dans les monts de Matmata a utilisé les données relatives à la végétation recueillies à partir des relevés floristiques effectués sur terrain. Par ailleurs, l'ensemble des espèces a été rattaché à ses hiérarchies botaniques en se référant aux Flores de la Tunisie (Pottier-Alapetite 1979, 1981), au Flore de l'Afrique du Nord (Maire, 1952-1987), au Catalogue des plantes vasculaires du Centre et du Sud tunisien (Le Houérou 1959) ainsi qu'au Catalogue synonymique commenté de la flore de Tunisie (Le Floc'h et al 2010). La richesse floristique dans un territoire est l'un des indicateurs de mesure les plus utilisés dans les études sur la diversité. Le nombre d'espèces qui peut être déterminé pour une unité de surface quelconque est un outil de base dans l'évaluation de cette richesse. Plus le nombre d'espèces est élevé, plus on a la chance d'inclure une plus grande diversité génétique, 
phylogénétique, morphologique, biologique et écologique (Lévèque et Mounolou 2008).

\subsection{La Richesse Spécifique Totale}

La Richesse Spécifique Totale désigne le nombre total d'espèces que comporte un peuplement végétal dans un écosystème donné (Ramade 2003 cité par Ngom et al 2013). Le tableau 6 présente cette richesse qui se traduit par le nombre moyen d'espèces pérennes (NEP) par type de végétation et par aire minimale $\left(32 \mathrm{~m}^{2}\right)^{6}$. L'examen de ce tableau montre un grand nombre d'espèces dans un espace relativement restreint. C'est au niveau des steppes à Rhanterium suaveolens et Artemisia campestris et celles à Artemisia campestris et Thymus capitatus que s'affichent les richesses les plus élevées avec une moyenne 34 et 31 espèces, respectivement. La présence dans ces steppes de plaine et d'épandages d'espèces bien adaptées aux sols sablo-limoneux ou limoneux profonds au dessus d'un substrat limono-gypseux et la proximité des écoulements des eaux superficiels lors des épisodes pluvieux, expliquent en partie cette richesse. Par ailleurs ces deux types de milieu sont souvent en mosaïque avec les cultures en sec d'où la présence de plusieurs espèces postculturales.

Toutefois, c'est au niveau des steppes à Stipa tenacissima que se présente la richesse la plus faible avec une moyenne de 15 espèces seulement. Cette pauvreté est aussi expliquée par un facteur édaphique. En effet, cette steppe s'étend le plus souvent au niveau des piémonts à éboulis ou sur les interfluves en majorité encroûtés et privés des écoulements superficiels. Très peu d'espèces pérennes accompagnent alors Stipa tenacissima, en particulier celles qui s'adaptent à ces conditions, le cas de Gymnocarpos decander et Helianthemum Kahiricum. La généralisation d'un voile sableux couvrant la surface du sol accentue le plus souvent la pauvreté de ces espaces en espèces végétales.

\footnotetext{
${ }^{6}$ Afin de rendre compte de la richesse floristique dans la zone d'étude et de comparer entre les différents types de milieu, le nombre d'espèces recensées a été ramené à une unité de surface homogène $\left(32 \mathrm{~m}^{2}\right)$ qui est l'aire minimale considérée dans tous les travaux en écologie végétale dans le milieu aride tunisien (Le Houérou 1959, 1969, Floret al 1978, Floret et Pontanier 1982, Hanafi 2008).
} 
Richesse spécifique totale (NEP) des principales formations végétales de la zone d'étude

\begin{tabular}{lc}
\hline \multicolumn{1}{c}{ Formation végétale } & NEP / 32 $\mathbf{m}^{\mathbf{2}}$ \\
\hline Matorral bas à Juniperus phoenicea et Rosmarinus officinalis & 25 \\
Matorral bas à Genista microcephala et Rosmarinus officinalis & 23 \\
Pseudo-steppe à Astragalus armatus et Calycotome villosa & 18 \\
Steppe à Seriphidium herba-alba et Haloxylon scoparium & 20 \\
Steppe graminéenne à Stipa tenacissima & 15 \\
Steppe à Gymnocarpos decander et Helianthemum kahiricum & 17 \\
Steppe à Rhanterium suaveolens et Artemisia campestris & 34 \\
Steppe à Artemisia campestris et Deverra tortuosa & 18 \\
Steppe à Artemisia campestris et Thymus capitatus & 31 \\
\hline & Moyenne \\
\hline & 22,3 \\
\hline & Source : Observations de terrain.
\end{tabular}

$\mathrm{Au}$ niveau du matorral méditerranéen présent dans la zone d'étude avec deux groupements, et malgré l'état généralement dégradé des ses espèces, la richesse spécifique est importante puisque le matorral à Juniperus phoenicea et Rosmarinus officinalis et celui à Genista microcephala et Rosmarinus officinalis présentent des moyennes de 25 et 23 espèces par aire minimale, respectivement. Ces valeurs sont d'autant plus importantes qu'elles dépassent la moyenne établie par Le Houérou (1995) pour l'ensemble des steppes tunisiennes soit 20,4 espèces. Elles confirment l'originalité de ces unités dans ce paysage steppique et peuvent être expliquées par la localisation de ce matorral hétérogène dans un espace de transition entre le milieu méditerranéen et celui subtropical désertique.

\subsection{La Richesse Spécifique Moyenne}

La richesse spécifique moyenne correspond au nombre moyen d'individus d'une même espèce par relevé pour un échantillon donné (Ngom et al 2013). Compte tenu des conditions bioclimatiques, édaphiques et anthropiques 
relativement particulières entre les milieux qui composent la zone d'étude, il a été nécessaire d'établir cet indicateur pour chacun de ces milieux et pour les principales espèces de la région (Tab. 7).

Tableau 7

Nombre moyen d'individus des principales espèces pérennes en fonction du milieu

\begin{tabular}{lccccc}
\hline Espèces & Montagne & Piémont & Plaine & $\begin{array}{c}\text { Fond } \\
\text { d'oued }\end{array}$ & Moyenne \\
\hline Juniperus phoenicea & 1,92 & 0,002 & 0 & 0 & 0,48 \\
Rosmarinus officinalis & 2,49 & 1,88 & 0 & 0 & 1,09 \\
Genista microcephala & 3,28 & 0,89 & 0 & 0 & 1,04 \\
Astragalus armatus & 3,86 & 4,34 & 6,29 & 3,92 & 4,60 \\
Calycotome villosa & 2,12 & 1,13 & 1,12 & 2,84 & 1,80 \\
Seriphidium herba-alba & 1,33 & 5,23 & 0,26 & 3,42 & 2,56 \\
Haloxylon scoparium & 0,22 & 3,91 & 2,66 & 3,51 & 2,58 \\
Stipa tenacissima & 3,69 & 6,75 & 2,62 & 1,12 & 3,55 \\
Gymnocarpos decander & 3,41 & 4,81 & 3,82 & 0,14 & 3,05 \\
Helianthemum & 3,32 & 5,12 & 3,79 & 0,27 & 3,13 \\
kahiricum & 0,12 & 1,28 & 7,33 & 6,12 & 3,71 \\
Rhanterium suaveolens & 0,2 & 0,92 & 6,19 & 7,93 & 3,81 \\
Artemisia campestris & 0,13 & 2,12 & 5,32 & 6,77 & 3,59 \\
Deverra tortuosa & 0 & 0,22 & 0,54 & 5,96 & 1,68 \\
Thymus capitatus & &
\end{tabular}

Source : Observations de terrain

A l'échelle de la zone d'étude (Fig. 6), c'est Astragalus armatus, qui affiche le nombre moyen d'individus le plus élevé par aire minimale soit 4,60. Cette espèce qui n'est devenue bien visible en milieu aride tunisien qu'à partir de la fin des années 1980, a montré des capacités à coloniser plusieurs types de milieu dans des conditions très différentes. En effet, la capacité de cette espèce ubiquiste à s'adapter à la rudesse du climat (forte chaleur, froid hivernal, manque d'eau...), aux différents types de sol même ceux les moins appropriés (sols minéraux bruts, encroûtés, avec voile sableux...) ainsi qu'aux activités humaines intensives 
(surpâturage), ont fait d'elle l'espèce la plus présente aujourd'hui en milieu aride tunisien. Hanafi $(2000,2008)$ a parlé du rôle qu'elle joue, de part son omniprésence, dans la tendance actuelle des steppes arides tunisienne vers l'homogénéité floristique, mais aussi de son rôle dans la conservation des équilibres écologiques en contribuant à limiter les problèmes liés à la désertification.

Le deuxième groupe d'espèces bien présentes dans la zone d'étude est formé par Artemisia campestris (3,81 individus), Rhanterium suaveolens $(3,71)$ et Stipa tenacissima $(3,55)$. Bien qu'elles colonisent des terrains différents, ces espèces indiquent toutes les trois les parcours en piémont et en plaine avec des sols relativement peu favorables à la mise en culture. Stipa tenacissima est essentiellement présente en piémont $(6,75$ individus) notamment au niveau des cônes d'éboulis et des interfluves aux sols calcaires. Quand à Rhanterium suaveolens et Artemisia campestris, elles sont très présentes au niveau des plaines sableuses et sablolimoneuses puisque les nombres respectifs de leurs individus dans ces milieux augmentent à 7,33 et 6,19. L'examen du tableau 7 montre aussi que la richesse spécifique moyenne dans la région est très influencée par les activités humaines. C'est le cas des espèces qui indiquent le surpâturage comme Stipa tenacissima, Astragalus armatus, Calycotome villosa ou qui indiquent le défrichement comme Deverra tortuosa ou Artemisia campestris. Cette dernière espèce est encore plus riche au niveau des épandages et des fonds d'oueds avec un nombre d'individus qui atteint 7,93.

Le dernier aspect qui peut être observé concerne le nombre moyen d'individus recensés pour les espèces de matorral à savoir Juniperus phoenicea, Rosmarinus officinalis et Genista microcephala. Ces espèces montrent une richesse moyenne relativement faible au niveau de la zone d'étude avec 0,$48 ; 1,09$ et 1,04 individus. Elles sont, toutefois, bien présentes en montagne ou leur richesse moyenne augmente à 1,92 ; 2,49 et 3,28 individus, respectivement. Malgré l'état dégradé de ce matorral, ces nombres moyens d'individus par espèce confirment la richesse globale des monts de Matmata avec ce mélange original entre espèces méditerranéennes et espèces steppiques. 


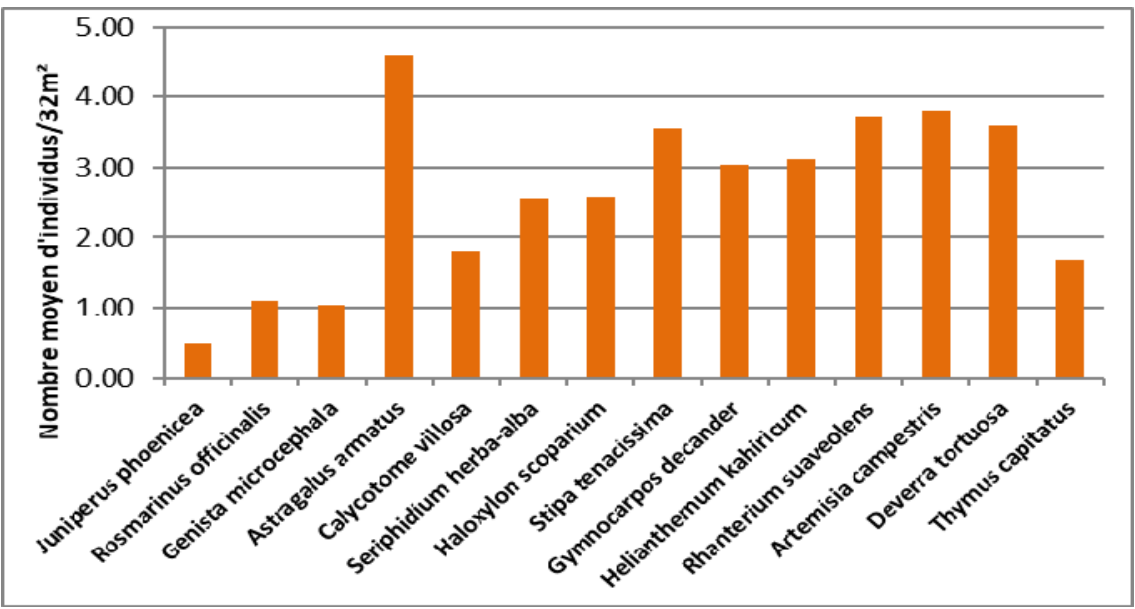

Fig. 6 : Richesse spécifique moyenne dans la zone d'étude.

Source : Observations de terrain

\subsection{Une importante richesse taxinomique dans un espace de transition}

La richesse floristique représente la liste de tous les végétaux de rang taxonomique divers (famille, genre, espèces, sous espèces et variété) qui peuplent un écosystème (Long 1974). L'examen des relevés effectués sur le terrain a permis de répartir les 82 espèces rencontrées en 32 familles (Tab. 8).

Compte tenu de la superficie relativement limitée de la zone d'étude, ce nombre d'espèces et surtout de familles s'avère être très important. L'analyse de la diversité relative des familles inventoriées dans la région montre que la famille des Asteraceae est la plus abondante renfermant 15 espèces suivies des Fabaceae avec 8 espèces et des Lamiaceae avec 7 espèces et ensuite les Brassicaceae avec 6 espèces. Le reste des familles sont présentes par moins de 5 espèces chacune. D'une manière générale, ce résultat confirme les connaissances en ce qui concerne la richesse spécifique en Tunisie Méridionale. En effet, cette région est considérée comme ayant la richesse aréale la plus élevée en Afrique du Nord. 
Tableau 8

Répartition des espèces sur les différentes familles de végétaux dans la zone d'étude

\begin{tabular}{lc|lc}
\hline \multicolumn{1}{c|}{ Famille } & Nombre d'espèces & \multicolumn{1}{c}{ Famille } & $\begin{array}{c}\text { Nombre } \\
\text { d'espèces }\end{array}$ \\
\hline Asteraceae & 15 & Chenopodiaceae & 1 \\
Fabaceae & 8 & Cleomaceae & 1 \\
Lamiaceae & 7 & Clusiaceae & 1 \\
Brassicaceae & 6 & Crassulaceae & 1 \\
Cistaceae & 5 & Cupressaceae & 1 \\
Liliaceae & 4 & Ephedraceae & 1 \\
Poaceae & 4 & Oleaceae & 1 \\
Geraniaceae & 4 & Plantaginaceae & 1 \\
Boraginaceae & 2 & Polygonaceae & 1 \\
Caryophyllaceae & 2 & Ranunculaceae & 1 \\
Solanaceae & 2 & Resedaceae & 1 \\
Zygophyllaceae & 2 & Rhamnaceae & 1 \\
Amaranthaceae & 2 & Rutaceae & 1 \\
Anacardiaceae & 1 & Scrophulariaceae & 1 \\
Apiaceae & 1 & Thymelaeaceae & 1 \\
Asclepiadaceae & 1 & Xanthorrhoeaceae & 1 \\
\hline
\end{tabular}

Source : Observations de terrain

Outre cette diversité spécifique, 1490 espèces sont présentes dans cette partie du pays, à laquelle s'ajoute une diversité génétique remarquable parfois au sein de la même espèce ou du même genre (Neffati et al 1999). C'est l'exemple du genre Astragalus qui est représenté dans la région par Astragalus armatus ssp. Tragacanthoides, Astragalus tenuifolius, Astragalus caprinus, Astragalus cruciatus et Astragalus gombiformis. Mis à part Astragalus armatus ssp. tragacanthoides qui a la capacité de coloniser plusieurs types de sol, l'ensemble de ces espèces préfèrent les sols sablo-limoneux à limoneux (Hanafi 2008). Le deuxième cas est celui du genre Helianthemum qui présente dans la région deux espèces; Helianthemum kahiricum et Helianthemum lippii. Cette dernière espèce, présente quand à elle, deux variétés dans la région à savoir Helianthemum lippii var. intricatum sur sols limono-gypseux et Helianthemum lippii var. sessiliflorum sur sols limono-sableux. 


\subsection{Une importante richesse en fonction de l'origine biogéographique des espèces}

Les monts de Matmata et leurs piémonts, constituent l'une des régions naturelles du Sud tunisien, les plus intéressantes sur le plan biologique et biogéographique (Le Floc'h 1973, Hanafi 2008). La position de la région entre les étages bioclimatiques aride supérieur et inférieur, la proximité du désert au sud et à l'ouest et de la mer méditerranée à l'est, l'importante trace de l'histoire géologique et humaine et l'absence de barrières géographiques empêchant la propagation des semenciers, sont tous des facteurs qui concourent à une importante diversité végétale de cette région. Le tableau 9 montre la répartition des espèces de la zone en fonction de leurs origines biogéographiques.

Selon ce tableau, le paysage végétal de la zone d'étude est dominé par des espèces méditerranéennes avec plus de $58 \%$ de l'ensemble du cortège floristique recensé dans la région. Elles sont suivies par les espèces saharo-arabiques dont la part est importante aussi de l'ordre de $26,8 \%$. Le reste des espèces (12 au total) sont réparties entre les origines saharienne, nord-africaine, cosmopolite et européenne, respectivement. Les deux dernières origines ne sont, en fait, représentées qu'avec une seule espèce chacune.

Tableau 9

Répartition des espèces végétales en fonction de leur origine biogéographique

\begin{tabular}{lcc}
\hline \multicolumn{1}{c}{ Origine biogéographique } & Nombre d'espèce & $\mathbf{\%}$ \\
\hline Méditerranéennes & 48 & 58,6 \\
Saharo-arabiques & 22 & 26,8 \\
Sahariennes & 6 & 7,3 \\
Nord-Africaines & 4 & 4,9 \\
Cosmopolites & 1 & 1,2 \\
Européennes méridionales & 1 & 1,2 \\
\hline Total & $\mathbf{8 2}$ & $\mathbf{1 0 0}$ \\
\hline Source : Aridhi 2016 d'après Le Houérou 1959, 1969, Pottier-Alapetite 1979, 1981
\end{tabular}


Afin de mieux comprendre cette diversité en rapport avec l'origine biogéographique des espèces, une comparaison a été établie entre trois sites de la zone d'étude choisis en fonction des critères liés au type de sol, au type de relief et aux conditions bioclimatiques. Il s'agit d'abord de Toujène et Zmerten. Ce sont deux sites localisés en montagne au relief calcaire. Ils sont dominés par un matorral rabougri avec un recouvrement moyen des espèces pérennes de l'ordre de $25 \%$ (Hanafi 2008). Environ $75 \%$ des espèces sont d'origine méditerranéenne et $60 \%$ d'entre elles sont des chaméphytes. Pas loin de ces deux premiers sites de montagne, se trouvent le troisième site qui se localise en plaine autour du village de Ksar Jedid. Il est envahi par un cortège floristique sur sols limono-sableux et caillouteux d'épandages, et dominé par une steppe composée d'espèces saharo-arabiques. La diversité est ici moins importante et le recouvrement végétal des espèces pérennes est, en général, plus faible se situant entre 10 et $15 \%$. D'après cette comparaison deux facteurs interviennent pour expliquer la différence entre ces deux types de stations. Il s'agit d'abord d'un facteur altitudinal qui favorise l'humidité et attire les pluies. Celles-ci sont beaucoup plus importantes en montagnes $(>200 \mathrm{~mm})$ qu'en piémonts et en plaines $(<150 \mathrm{~mm})$. Les facteurs humains sont aussi pour beaucoup dans cette situation puisque la collecte des eaux pluviales au niveau des impluviums en amont offre aux secteurs de montagne un excédent en humidité qui améliore la diversité végétale. En plaine, la situation est totalement différente puisqu'en plus du manque d'eau, la forte pression humaine sur les végétaux et les sols (surpâturage, défrichement...) contribue fortement à la dégradation de la diversité.

\subsection{Une importante richesse en fonction des caractéristiques} biologiques des espèces

Le type biologique d'une plante est la résultante, sur la partie végétative de son corps, de tous les processus biologiques y compris ceux qui sont modifiés par le milieu pendant la vie de la plante et ne sont pas héréditaires (Polumin 1967). Les espèces, du point de vue biologique, sont avant tout, organisées pour traverser la période critique du cycle saisonnier, qui peut être l'hiver à cause du froid ou l'été à cause de la sécheresse. Parmi les nombreux systèmes proposés de classification 
des types biologiques, celle élaborée par Raunkiaer (1918) et modifiée par Braun-Blanquet (1932) et qui classe les végétaux en cinq types à savoir les Phanérophytes, les Chamaephytes, les Hémicryptophytes, les Géophytes et les Thérophytes. Le tableau 10 montre la répartition des espèces recensées dans la zone d'étude en fonction des ces cinq types.

Tableau 10

Spectre biologique brut des espèces végétales de la zone d'étude.

\begin{tabular}{lcc}
\hline Type biologique & Nombre d'espèce & $\mathbf{\%}$ \\
\hline Chaméphytes & 38 & 46,3 \\
Thérophytes & 19 & 23,2 \\
Hémicryptophytes & 9 & 11,0 \\
Géophytes & 8 & 9,8 \\
Phanérophytes & 8 & 9,8 \\
\hline TOTAL & $\mathbf{8 2}$ & $\mathbf{1 0 0}$ \\
\hline
\end{tabular}

Source : Aridhi 2016.

Le spectre biologique brut des espèces inventoriées confirme la présence des formations steppiques puisque il montre une importance relative des Chaméphytes avec plus de $46 \%$ suivies par les Thérophytes avec plus de $23 \%$ (Tab. 10). Les Chaméphytes sont considérés comme étant les espèces pérennes les mieux adaptées aux basses températures hivernales et à l'aridité (Floret et al 1978).

Cette importance est aussi confirmée par le spectre biologique net (Tab. 11) qui montre toujours une grande dominance des Chaméphytes avec plus de $58 \%$. L'observation détaillée de ce type biologique montre que les Chaméphytes méditerranéennes représentent $36 \%$ alors que celles steppiques se limitent à $22 \%$. Ce résultat issu des observations de terrain est expliqué par l'importante influence de la topographie et du bioclimat aride supérieur avec son humidité plus élevée et son froid hivernal sur le nombre et la dominance des espèces méditerranéennes.

Quant aux Phanérophytes et malgré leur faible importance relative $(9,8 \%)$, elles présentent aussi une grande importance de point de vue dominance puisque leur part s'élève à 16\% dans le spectre biologique net. Les visites de terrain on montré que la plupart de ces espèces qui caractérisent le matorral sont le plus souvent très rabougries. C'est 
l'exemple de Juniperus phoenicea dont la hauteur dépasse rarement les 2 mètres et le périmètre de sa partie aérienne les 4 mètres.

Tableau 11

Spectre biologique net des espèces végétales de la zone d'étude.

\begin{tabular}{lcc}
\hline Type biologique & $\begin{array}{c}\text { Coeff } \mathbf{d}^{\prime} \text { 'abondance } \\
\text { dominance }^{7}\end{array}$ & $\mathbf{\%}$ \\
\hline Chaméphytes & 39,8 & 58,0 \\
Thérophytes & 8,4 & 12,2 \\
Hémicryptophytes & 5,2 & 7,4 \\
Géophytes & 4,4 & 6,4 \\
Phanérophytes & 10,8 & 16,0 \\
\hline TOTAL & $\mathbf{6 8 , 6}$ & $\mathbf{1 0 0}$ \\
\hline
\end{tabular}

Source : Observations de terrain

Ces données offrent ainsi à cette région un paysage végétal assez original et véritablement azonal qui contribue à la richesse végétale et à la diversité paysagère de la zone d'étude.

\subsection{Endémisme et rareté des espèces; une force ou une vulnérabilité?}

L'examen du cortège floristique de la zone d'étude a montré la présence de quelques espèces endémiques ou rares (Tab. 12 et Fig. 5 supra). Ce tableau confirme les différentes formes de diversité végétale de la région puisqu'il ajoute une donnée aussi bien importante qu'originale. En effet, la zone d'étude renferme 9 espèces endémiques dont une espèce endémique de Matmata à savoir Rosmarinus officinalis var. troglodytarum. Ce nombre est relativement important pour une superficie limitée. Il faut, toutefois, relativiser cette constatation puisque les 8 autres espèces sont endémiques de tout l'Afrique du Nord ou du

7 Le Coefficient d'abondance-dominance est un indice de la dominance d'un type biologique par rapport aux autres. Il est utilisé dans les relevés de Braun-Blanquet et va de 0,1 pour une espèce simplement présente à 5 pour une espèce en peuplement pur (Braun-Blanquet, 1932). Dans ce travail le coefficient présenté dans le tableau 9 est une moyenne de l'ensemble des coefficients obtenus à partir des relevés effectués sur terrain. 
Sud de la Méditerranée. Seules Artemisia campestris subsp. Cinerea et Teucrium alopecurus sont endémiques de la Tunisie.

Tableau 12

Les espèces endémiques et rares de la zone d'étude

\begin{tabular}{ll}
\hline \multicolumn{1}{c}{ Espèce } & \multicolumn{1}{c}{ Endémisme ou rareté } \\
\hline Artemisia campestris subsp. cinerea & Endémique de la Tunisie \\
\hline Asparagus albus & Rare \\
\hline Astragalus armatus ssp. tragacanthoides & Endémique Nord-Africaine \\
\hline Deverra tortuosa & Endémique Nord-Africaine \\
\hline Lygeum spartum & Endémique Sud méditerranéenne \\
\hline Olea europaea & Rare \\
\hline Ononis narix ssp. falcata & Endémique \\
\hline Anarrhinum brevifolium & Rare \\
\hline $\begin{array}{l}\text { Rosmarinus officinalis var. } \\
\text { troglodytarum }\end{array}$ & Endémique de Matmata \\
\hline Scilla villosa & Endémique Egypte/Libye/Tunisie \\
\hline Teucrium alopecurus & Endémique de la Tunisie \\
\hline Thymus hirtus var. algeriensis & Endémique Nord-Africaine \\
\hline
\end{tabular}

Source : Observations de terrain

Le taux d'endémisme est par conséquent très élevé puisqu'il atteint $11 \%$ ce qui montre l'importance de ce paysage végétal. Mis à part ces espèces endémiques, le paysage présente quelques espèces de plus en plus rares dans le paysage. C'est le cas notamment d'Asparagus albus, Olea europaea, et Anarrhinum brevifolium qui se font de plus en plus rares dans la région. Bien que ces espèces ne sont pas endémiques mais les rencontrer sur terrain est aujourd'hui une mission presque impossible. Cette situation est d'autant plus critique qu'Anarrhinum brevifolium dominait avec Zygophyllum album une association végétale caractéristique des piémonts limono-gypseux arides tunisiens (Le Houéou 1959, 1969). Aujourd'hui seule Zygophyllum album existe au niveau de ces terres associée à d'autres espèces qui ont probablement remplacé Anarrhinum brevifolium comme Ononis natrix ssp. falcata, Moricandia arvensis ou encore Helianthemum lippii var. intricatum (Hanafi, 2000). 
L'ensemble de ces caractéristiques des espèces de Matmata est certainement un atout pour la région, mais peut être aussi considéré comme un indicateur écologique de dégradation de ces écosystèmes originaux (Hanafi et Jauffret, 2008). Le suivi à long terme de ces espèces et ces écosystèmes est plus que nécessaire pour pouvoir les conserver et participer à maintenir la diversité dans cette région. Dans cette région, plusieurs travaux de recherche précédents ont auparavant traité la question de la dégradation de la végétation aride (Le Houérou 1959, Le Floc'h 1973, Hanafi 2000). La plupart de ces travaux ont évoqué la difficulté de faire des évaluations des états de la végétation notamment en rapport avec les conditions naturelles (sécheresses) et humaines (pâturage, défrichement...). Mais ils ont bien montré les faibles capacités de résilience des écosystèmes arides du Sud tunisien. La dégradation débute en général par une altération de la végétation et une modification de la composition floristique. Les espèces les plus utilisées se raréfient progressivement et disparaissent. Ensuite ou parallèlement, le couvert végétal s'éclaircisse et la production de biomasse diminue. Les capacités de reproduction et de régénération de la végétation se réduisent de plus en plus (Genin et al 2006) et les espèces qui se raréfient ou qui disparaissent sont souvent difficilement récupérables.

\section{Le matorral de Matmata, vecteur de développement}

Selon Amri (2002) "L'enclavement est un processus par lequel une population se trouve isolée de l'ensemble de la société à laquelle elle appartient, en raison de l'absence ou de la détérioration des moyens et des réseaux de communication. Il touche la capacité productive de la région au sens strictement économique mais touche aussi les secteurs improductifs comme la santé, la scolarisation et les routes qui servent à relier une région au reste du pays et l'inclure dans la dynamique du développement». Bien que les monts de Matmata bénéficient d'une infrastructure relativement acceptable par rapport à d'autres arrière-pays tunisiens, ils demeurent enclavés sur le plan économique puisqu'ils sont toujours en marge de l'économie nationale. Leur conditions naturelles contraignantes, augmentent leur fragilité et leur marginalité et la détérioration des conditions socioéconomiques des populations. 
C'est dans cette perspective que s'impose aujourd'hui un développement de ces régions qui devrait non seulement être innovant mais qui devrait aussi permettre à la population locale de limiter la précarité de leurs économies. Le matorral de Matmata constitue une des ressources de la région qui présente un potentiel important à valoriser. Bien que cette ressource ait, depuis longtemps, été intégrée dans système de production traditionnel basé sur le pastoralisme extensif et l'agriculture derrière les jessour, il est aujourd'hui possible de la valoriser au moins sous deux «nouvelles » formes à savoir l'exploitation médicinale et thérapeutique des plantes et la valorisation éco-touristique de ce paysage original.

\subsection{Un matorral aux multiples vertus}

La plupart des plantes méditerranéennes ont été utilisées, depuis l'Antiquité, par les populations dans une médecine traditionnelle qui s'est souvent avérée efficace et que la science a prouvé. Les populations locales y compris celle de Matmata ont, au fil du temps, acquis un important savoir-faire local en matière de valorisation médicinale et thérapeutique d'une bonne partie des plantes locales. Plusieurs espèces possèdent aussi une importante valeur nutritive non seulement pour les hommes mais aussi pour les animaux domestiques. C'est le cas de plusieurs espèces caractérisant le matorral de Matmata qui sont souvent peu ou pas utilisées à ces fins et dont une valorisation moderne pourrait constituer une alternative intéressante pour développer les économies familiales. Nous citons ici quelques exemples qui pourraient être d'une grande utilité pour les habitants.

Le premier cas est celui de Juniperus phoenicea ou le Genévrier de Phénicie. Ce phanérophyte rabougri qui domine entre les localités de Toujène et Béni Khédache pourrait être exploitée de plusieurs manières. En effet, cette espèce est connue dans la médecine traditionnelle au Maghreb pour ses effets diurétiques, stomachiques et digestifs. Les tanins que les baies contiennent forment une épice appréciée par certains car elle facilite la digestion et permet de combattre les gaz intestinaux. Cette plante a aussi un potentiel antiseptique et dépuratif. Elle est également utilisée pour soigner l'asthme, l'arthrite, la goutte et toutes les maladies 
rhumatismales. Elle est enfin utilisée pour combattre le diabète et abaisser les taux du glucose sanguin (Bertaudière-Montes et al 2004).

Le deuxième cas est celui de Rosmarinus officinalis ou le romarin. Il s'agit d'une chaméphyte méditerranéenne aux multiples usages. C'est une plante qui peut d'abord être consommée. En effet, les branches feuillues du romarin s'utilisent dans la cuisine méditerranéenne de préférence fraîches, mais peuvent également se conserver après avoir été séchées. Les fleurs ont une saveur plus douce et se consomment crues, saupoudrées pour parfumer un plat ou un dessert. Les branches s'emploient généralement comme aromate par infusion dans plusieurs plats comme les soupes, les sauces et les grillades, etc. Le romarin est également un produit fréquemment utilisé en parfumerie. Son essence qui contient du bornéol, du cinéol, du camphène et du pinène est obtenue par la distillation des branches fleuries (Minker 2013). Enfin, on lui attribue de nombreuses vertus phytothérapeutiques. En effet, le romarin permet d'activer les fonctions digestives, en particulier le travail de la vésicule biliaire. C'est aussi un antimycosique et un antibactérien. Il a également un effet sur le système nerveux puisque l'administration d'huile de romarin, à la fois par inhalation et par voie orale, stimule l'activité du système nerveux central et respiratoire. Le romarin serait donc recommandé pour traiter les divers cas d'asthénie. Il a aussi des effets sur la circulation sanguine puisqu'il a été prouvé scientifiquement que l'utilisation d'huile de romarin dans un bain stimule la circulation dermique et améliore l'hémodynamique pour les problèmes d'occlusion artérielle (Fleurentin 2013).

Le troisième cas est celui de Seriphidium herba-alba ou l'Armoise blanche. Selon Ben Haj Jilani et al (2014) et Aichi-Yousfi et al (2016), cette espèce présente un pouvoir allélopathique puisque son huile essentielle est un inhibiteur de la germination des graines de certaines mauvaises herbes comme Sinapis arvensis L. et Phalaris minor Retz. Dans la médecine traditionnelle, l'armoise blanche a été reconnue par les populations pastorales et nomades pour ses vertus purgatives. On l'utilise notamment comme vermifuge chez les ovins. L'infusion de cette plante est assez employée pour soulager les maux gastro-intestinaux. En Tunisie, elle est également utilisée pour les maladies du tractus digestif et comme un traitement antidiabétique. 
Plusieurs autres plantes qui composent le cortège floristique de Matmata, sont très intéressantes et dont l'utilisation rationnelle pourrait constituer une nouvelle source de revenus à la population locale. C'est le cas de Ruta chalepensis (Fijel) connue pour avoir des propriétés antispasmodiques, anti-inflammatoires, antibiotiques, insectifuges, antifongiques et antiseptiques. C'est le cas aussi de Capparis spinosa (Le Câpre) qui, selon Ben Mansour et al (2014) et Aichi-Yousfi et al (2016), est connue pour être un antioxidant. La consommation de ses boutons floraux sert notamment à stimuler l'appétit et ses racines seront utilisées en médecine traditionnelle (en infusion ou en décoction) comme un diurétique ou encore pour lutter contre l'arthrite et le rhumatisme.

\subsection{Le matorral de Matmata: un potentiel éco-touristique}

Les monts de Matmata sont dotés de paysages rares et de potentialités écologiques importantes caractérisées par une grande diversité biologique. Ces potentialités constituent des éléments de diversité, de richesse et des facteurs d'attraction pour lesquels les sociétés rurales ont développé des savoirs spécifiques permettant la valorisation des ressources locales. Le matorral de Matmata, est une des ces ressources qui constitue aujourd'hui un important réservoir de biodiversité. Selon la déclaration universelle de l'UNESCO sur la biodiversité en 2002, cette dernière est considérée comme "un patrimoine commun de l'humanité dont le respect constitue une valeur fondamentale de l'humanité. C'est une forme de diversité de la vie sur terre définit par la relation qui existe entre la diversité culturelle et la biodiversité qui sont toutes les deux des valeurs pour le très long terme ». Il existe, par conséquent, un lien inextricable entre la diversité biologique et la diversité culturelle (Loh et Harmon 2005).

La biodiversité doit être perçue sous l'angle de la diversité humaine, car les différentes cultures et les individus d'origines sociales différentes appréhendent et saisissent la biodiversité de diverses façons en raison de leur propre héritage et de leurs expériences. De ce fait, la diversité culturelle est le reflet de la biodiversité. A partir de cette idée originale, plusieurs initiatives ont eu lieu dans le monde en essayant de valoriser la biodiversité pour en tirer un maximum de profits économiques et notamment touristiques. C'est le cas des différents parcs 
forestiers qui ont vu le jour par exemple en France depuis 1968 et qui ont permis aux populations riveraines d'exploiter les richesses biologiques et écologiques dont ils disposent, exp : Le parc naturel régional Scarpe-Escaut dans le Nord-Pas-de-Calais-Picardie, le parc ornithologique des Landes de Gascogne en région Nouvelle-Aquitaine, Le parc du Verdon dans les Alpes-de-Haute-Provence et le Var, etc. (Lévèque et Mounolou 2008).

C'est dans cette perspective qu'il est très important aujourd'hui pour les acteurs locaux et régionaux dans le Matmata de réfléchir à développer une activité éco-touristique qui pourrait valoriser le réservoir de biodiversité dont ils disposent, non seulement en tant que richesse environnementale originale, mais aussi en tant qu'héritage culturel accumulé par les populations locales et qui mérite d'être connu et visité. La richesse environnementale de cette région pourrait aussi être mieux «vendus » aux visiteurs avertis si on lui associe les autres acquis et savoir-faire patrimoniaux en matière agricole (jessour...), culturelle (Produits de la laine, cuisine locale, plantes médicinales...) et architecturale (habitat troglodyte, Ksour...) qui se concentrent dans cette région.

\section{Conclusion}

La cartographie de la végétation des monts arides de Matmata entre Toujène et Béni Khédache a montré l'importante diversité des types de végétation expliquée par le rôle essentiel des conditions naturelles et de l'homme dans ce milieu contraignant. Malgré la dégradation quantitative de cette ressource, le cortège floristique demeure riche surtout en espèces méditerranéennes et saharo-arabiques avec quelques espèces rares et endémiques et qui nécessitent aujourd'hui une attention particulière. Compte tenu de l'absence dans cet arrière-pays aride d'un secteur moteur de développement (agriculture familiale traditionnelle, faibles investissements dans le commerce de l'artisanat...), il est aujourd'hui plus que nécessaire de promouvoir des activités innovantes, entre autres, autour de la ressource végétale (valorisation médicinale, apicole et éco-touristique) qui permettrait de diversifier les revenus des familles rurales et de participer à limiter l'enclavement de cette région. 
Il faut, toutefois, ne pas oublier que le matorral dans cette région, originale comme elle l'est, est considérée comme un patrimoine naturel qui nécessite d'être conservé. En effet, ce couvert végétal constitue dans cette zone un élément fondamental du fonctionnement des écosystèmes et un support important d'activités humaines. Cette végétation présente des caractéristiques d'adaptation climatiques remarquables dans un écosystème fragile (rareté et variabilité des pluies, températures élevés, vents chauds et secs). La diversité végétale dans cette région est un patrimoine et une richesse à transmettre. Donc préserver la biodiversité, c'est préserver la richesse de la nature où certaines plantes rendent des services considérables aux sociétés humaines. Sa valorisation devrait passer par la mise en place d'un modèle de développement local qui garantit une exploitation rationnelle non seulement de la végétation mais de toutes les autres ressources locales.

\section{Remerciements}

Ce travail a bénéficié du financement de l'Agence Nationale française de la Recherche (ANR) dans le cadre du projet franco-maghrébin MedInnLocal (ANR-12-TMED-0001): "Innovation autour des spécificités locales dans les arrière-pays méditerranéens ». Les auteurs tiennent à remercier tous les participants à ce projet et notamment l'équipe tunisienne qui a participé à la réalisation des travaux de terrain à savoir messieurs Amor Mokhtar GAMMAR, Khaled ABAZA, Hédi ABDALLAH et Mourad LARIBI.

\section{BIBLIOGRAPHIE}

Abaab A., 1986, Mutations socio-économiques de la Jeffara orientale (Sud tunisien). In "Désert et montagne au Maghreb", hommage offert à Jean Dresh, ROMM (41-42) : 327-338.

Abichou H., 2009, La valorisation du patrimoine vecteur de développement local durable : quelles retombées économiques et quel dispositif institutionnel ?: Cas $d u$ sud-est tunisien. Thèse Doct, Université Montpellier 1. 272p.

Aichi-Yousfi H., Meddeb E., Rouissi W., Hamrouni L., Slim R., Rejeb N., Gharbi-Gammar Z., 2016, Phenolic composition and antioxidant activity 
of aqueous and ethanolic leaf extracts of six Tunisian species of Genus Capparis - Capparaceae. Industrial Crops and Products, Vol. 92, pp : 218-226. Amri L., 2002, La femme rurale dans l'exploitation familiale, Nord-ouest de la Tunisie : pour une sociologie des ruptures. Ed. L'Harmattan., Paris., 538p.

Aridhi O., 2016, Cartographie de la végétation et étude des formes d'usage de la laine de mouton et leur impact sur les systèmes de production familiaux dans les jebels Matmata centraux (sud-est tunisien). Mémoire de Mastère en Biogéographie, FLAHM, Univ. de Manouba, 114p.

Ben Fredj T., 2012, La Jeffara septentrionale : étude de l'évolution géomorphologique au cours du Quaternaire. Thèse Doct., Univ. De Tunis, FSHST.

Ben Haj Jilani I., Chebil S., Khiari R., Melki I. Limam-Ben Saad S., DaoudBouattour A. et Ghrabi-Gammar Z., 2014, Allelopathic potential of some essential oils vis-à-vis three noxious weed species invading cereals. International Journal of Agronomy and Agricultural Research (IJAAR), 4:77-97.

Ben Kehia H., Claude J. et Ouessar M., 2002, Le risque de dégradation des ressources naturelles dans la zone de piémont du bassin versant de l'Oued Hallouf (région de Jeffara, Tunisie du Sud-Est). In: Oueslati A., KARRAY M. R. (éds.) Actes des 2èmes journées d'étude du CGMED « risques naturels et environnement ", FSHST, pp : 27-35.

Ben Mansour R., Ben Haj Jilani I., Bouaziz M., Gargouri B., Elloumi N., Attia H., Ghrabi-Gammar Z., et Lassoued S., 2014, Phenolic contents and antioxidant activity of ethanolic extract of Capparis spinosa. Cytotechnology. 68. 10.1007/s10616-014-9764-6.

Ben Ouezdou H., 2001, Découvrir la Tunisie du Sud: de Matmata à Tataouine. Ksour, Jessour et Troglodytes. Tunis, 80p.

Benzecri J-P., 1964, Sur l'analyse factorielle des proximités. Publ. Inst. Stat. Univ. Paris, 1 (14) : 165-180.

Bertaudière-Montès V., Montes N. et Giraud M., 2004, Le Genévrier. Actes Sud (Ed.), 96p.

Bonvallot J., 1986, Tabias et jessour du Sud tunisien. Agriculture dans les zones marginales et parade de l'érosion. Cah. ORSTOM, Sér. Pédol., vol. XXII, (2) : 163-171.

Boukhris M. et Lossaint P., 1972, Spécificité biogéochimique des plantes gypsophiles de Tunisie. Oecol. Plant. 7 (1) : 45-68.

Braun-Blanquet J., 1932, Les groupements végétaux de la France méditerranéenne. Ed. C. N. R. S. Paris, 279p.

Bry X., 1996, Analyses factorielles multiples. Economia (Ed.), poche techniques quantitatives. Paris, 112p. 
Coudé-Gaussen J., 1991, Les poussières sahariennes. Cycle sédimentaire et place dans les environnements et paléo environnements désertiques. (Ed.) John Libbey Eurotext, $485 \mathrm{p}$.

Derouiche R., 1997, Contribution à l'étude par modèle numérique de l'impact des aménagements de C.E.S. sur la recharge de la nappe de Zeuss-Koutine. Diplôme Ingé. en Agronomie, INAT, Tunis, 70p, annexes.

Emberger L., 1955, Une classification biogéographique des climats. Travaux Lab. Bot. Zool., Fac. Sci. Serv. Bot. Montpellier (7) : 3-43.

Escadafal R., 1979, Contribution à l'étude des ressources en sol de la plaine des Ababsas (Médenine). Étude $n^{\circ} 542$, Division des sols, Tunis, 49p., annexes, 2 cartes $h . t$.

Escofier B. et Pages J., 2002, Analyses factorielles simples et multiples. Objectifs, méthodes et interprétation. DUNOD (Ed.), 3ème édition, 2ème cycle, école d'ingénieurs, Paris, 284p.

Ferchichi A., 1996, Étude climatique en Tunisie présaharienne. Options Médit. (3) : 46-53.

Fleurentin J., 2013, Du bon usage des plantes qui soignent. Ouest-France (Ed.), $384 \mathrm{p}$.

Floret C., Le Floc'h E., Pontanier R. et Romane F., 1978, Modèle écologique en vue de la planification et de l'aménagement agro-pastoral des régions arides: Application à la région de Zougrata. ORSTOM (Ed.) ; 75 p.

Floret C. et Pontanier R., 1982, L'aridité en Tunisie présaharienne : climat, sol, végétation. Travaux et documents de l'ORSTOM, $\mathrm{n}^{\circ} 150,544 \mathrm{p}$.

Genin D., Hanafi A. et Ciladella N., 2006, L'agriculture dans la Jeffara : entre permanences et bouleversements, quelle place dans la reproduction des systèmes ruraux? In Genin D., Guillaume H., Ouessar M., Ouled Belgacem A., Romagny B., Sghaier M. et Taamallah H. (Eds.) : "Entre désertification et développement, la Jeffara tunisienne ", IRD/Cérès/IRA (Ed.), pp : 163-179.

Guillaume H., Romagny B., Fetoui M., Nouri H., Palluault S. et Hajji A., 2003, Sociétés, dynamiques d'occupation du milieu et compétitions sur les ressources naturelles dans la Jeffara tunisienne. Rapport scientifique $d u$ thème 2 du programme Jeffara, IRA, Médenine (Tunisie), 248p.

Hanafi A., 2008, Végétation steppique et systèmes de production agro-pastoraux au nord de la Jeffara tunisienne: Recherches sur les relations dynamiques. Thèse Doct, FSHST, Univ. de Tunis; 289p.

Hanafi A. et Jauffret S., 2008, Are long-term vegetation dynamics useful in monitoring and assessing desertification processes in the arid steppe, southern Tunisia. Journal of Arid Environments 72: 557-572. 
Le Floc'h E., 1973, Étude des parcours du Sud tunisien. I- Carte phyto-écologique de Oglat Merteba et Mareth. Annales de l'INRAT, vol. 46, fasc. 5, 96p., cartes $1 / 100.000$, annexes.

Le Floc'h E., Boulos L. et Véla E., 2010, Catalogue Synonymique Commenté De La Flore De Tunisie. Banque Nationale de Gènes de la Tunisie (Ed), MEDD, ISBN : 978-9938-9508-0-9, Tunisie, 500p.

Le Houérou H-N., 1959, Recherches écologiques et floristiques sur la végétation de la Tunisie méridionale. Univ. Alger. Institut de Recherches Sahariennes, Alger, Mémoire h.s. 2 tomes, 510p., 54 tab., 4 cartes, 1 pochette.

Le Houérou H-N., 1969, La végétation de la Tunisie steppique (avec références au Maroc, à l'Algérie et à la Libye). Annales de l'INRAT, vol. 42, fasc. 5, Tunis, $622 \mathrm{p}$. annexes, carte couleur -h.t.

Le Houérou H-N., 1995, Bioclimatologie et biogéographie des steppes arides du Nord de l'Afrique : diversité biologique, développement durable et désertisation. Options Méditerranéennes : Série B. Etudes et Recherches ; n. 10, Le Houérou H-N. (éd.) / CIHEAM, Montpellier, 396p.

Lévèque C., 2001, Ecologie. De l'écosystème à la biosphère. Masson Sciences. Dunod, Paris. 502p.

Lévèque C. et Mounolou J.C., 2008, Biodiversité. 2ème édition. Masson Sciences. Dunod, Paris. 248p.

Loh J. et Harmon D., 2005, A global index of biocultural diversity. Ecological Indicators, Vol. 5, (3) : 231-241.

Long G. 1974, Diagnostic phytoécologique et aménagement du territoire. I : Principes généraux et méthodes. Masson, Paris. 1 vol. 252p.

Maire R., 1952 - 1987, Flore de l'Afrique du Nord. Encyclopédie Biologique ; Vols. 1-16, Ed. Paul Lechevalier. Tela Botanica.

Minker C., 2013, 200 plantes qui vous veulent du bien. Larousse (Ed.), 448p.

Mtimet A., 1994, Contribution à l'étude pédologique des limons de Matmata (Sud Tunisie). Sols de Tunisie. Bulletin de la Direction des sols n 15, 341p.

Neffati M., Ghrabi Gammar Z., Akrimi N. et Henchi B., 1999, Les plantes endémiques de la Tunisie. Flora Mediterranea, vol. 9, p : 163-174.

Ngom D., Fall T., Sarr O., Diatta S. et Akpo L., 2013, Caractéristiques écologiques du peuplement ligneux de la réserve de biosphère du Ferlo, Sénégal. Journal of Applied Biosciences 65 : 5008 - 5023, ISSN 1997-5902. 
Ouessar M. et Yahyaoui H., 2006, Les ressources en eau. In Genin D., Guillaume H., Ouessar M., Ouled Belgacem A., Romagny B., Sghaier M. et Taamallah H. (Eds.) : "Entre désertification et développement, la Jeffara tunisienne ", IRD / Cérès/IRA (Ed.), pp : 47-56.

Polumin N., 1967, Eléments de géographie botanique. Ed. Gauthiers Willars. Paris, pp : 30-35.

Pottier-Alapetite G., 1979, Flore de la Tunisie Angiospermes - Dicotylédones. Tome 1 : Apétales - Dilapétales. Ed. Ministère de l'Enseig. Sup. et Ministère de l'Agri. Tunis, 651p.

Pottier-Alapetite G., 1981, Flore de la Tunisie Angiospermes - Dicotylédones. Tome 2: Gamopétales. Ed. Ministère de l'Enseig. Sup. et Ministère de l'Agri. Tunis, pp. 655-1190.

Raunkiaer C., 1918, Recherches statistiques sur les formations végétales. Biologics Meddeleser Kongelige Danske Videnskabernes Selskab 1:1-80.

Romagny B., Guillaume H., Ben Ouezdou H., Palluault S. et Sghaier M., 2003, Les enjeux de la gestion des déficits en eau dans la Jeffara (Sud-est tunisien) : état des lieux et perspectives. Colloque "Gouvernance de l'eau et développement durable », Sousse, 9-10 oct., 20p.

Taamallah H. et Ben Kehia H., 2006, Les caractéristiques géomorphologiques et les sols. In Genin et al (éd.): "Entre désertification et développement : la Jeffara tunisienne » Tunis (TUN) ; Tunis : IRD ; Cérès, 351 p.

Taamallah H., Genin D., Guillaume H., Hajji A., Ouled Belgacem A., Picouet M. et Romagny B. (Coord.), 2003, Environnement et société dans la Jeffara : un état des lieux. Rapport scientifique de synthèse, Programme Jeffara, IRA/IRD, pp : 21-68. 